\title{
A Systematic Algorithm for the Synthesis of Multiplierless Lattice Wave Digital Filters
}

\author{
Juha Yli-Kaakinen and Tapio Saramäki \\ Tampere University of Technology \\ Finland
}

\section{Introduction}

Among the best structures for implementing recursive digital filters are lattice wave digital (LWD) filters (parallel connections of all-pass filters). They are characterized by many attractive properties, such as a reasonably low coefficient sensitivity, a low roundoff noise level, and the absence of parasitic oscillations. This book chapter describes an efficient algorithm for the design of multiplierless LWD filters in the following three cases. In the first case, the overall filter is constructed as a cascade of low-order LWD filters. As a consequence, the number of bits required for both the data and coefficient representations are significantly reduced compared with the conventional direct-form LWD filter. In the second case, approximately linear-phase LWD filters are constructed as a single block because it has been observed that in this case the use of a cascade of several filter blocks does not provide any benefits over the direct-form LWD filter design. The third case concentrates on the design of special recursive single-stage and multistage $N$ th-band decimators and interpolators providing the sampling rate conversion by the factor of $N$. For this filter class, the decimation and interpolation filter in the single-stage design (the $k$ th decimation and interpolation filter in the multistage design, where $N$ is factorizable as a product of $K$ integers as $\left.N=N_{1} N_{2} \cdots N_{K}\right)$ is characterized by the fact that it can be decomposed into parallel connection of $N\left(N_{k}\right)$ polyphase components that are obtainable from cascades of first-order all-pass filters by substituting for each unit delay $N\left(N_{k}\right)$ unit delays.

The coefficient optimization is performed using the following three steps. First, an initial infinite-precision filter is designed such that it exceeds the given criteria in order to provide some tolerance for coefficient quantization. Second, a nonlinear optimization algorithm is used for determining a parameter space of the infinite-precision coefficients including the feasible space where the filter meets the given criteria. The third step involves finding the filter parameters in this space so that the resulting filter meets the given criteria with the simplest coefficient representation forms. The proposed algorithm guarantees that the optimum finiteprecision solution can be found for the multiplierless coefficient representation forms. Filters of this kind are very attractive in very large-scale integration implementations because the realization of these filters does not require the use of very costly general multiplier elements. Several examples are included to illustrate the benefits of the proposed synthesis scheme as well as the resulting filters. 


\section{Lattice Wave Digital Filters}

One of the best structures for implementing recursive digital filters are the lattice wave digital (LWD) filters (Fettweis, 1986; Fettweis et al., 1974; Gazsi, 1985; Wanhammar, 1998) that are related to certain analog prototype networks. The number of multipliers required in the implementation is directly the filter order, unlike in some other implementation forms, such as in the canonical direct-form realizations requiring approximately twice the number of multipliers.

An LWD filter consists of a parallel connection of all-pass filters. These all-pass subfilters can be realized by using first- and second-order sections as basic building blocks. The resulting filter structures are highly modular, thereby making them suitable for very large-scale integration (VLSI) implementations (Milić \& Lutovac, 1999; Saramäki \& Ritoniemi, 1993). All-pass subfilters are also the basic building blocks of recursive half-band filters (Ansari \& Liu, 1983; Gazsi, 1985), Hilbert transformers (Brophy \& Salazar, 1975; Regalia, 1993; Saramäki \& Renfors, 1995), filters approximately providing an arbitrary linear-phase phase response or an arbitrary phase delay in the given passband (Saramäki \& Renfors, 1995), several efficient recursive filter-bank classes (Bregović, 2003; Saramäki \& Bregović, 2002; Vollmer \& Kopmann, 2002), and recursive Nth-band filters (Renfors \& Saramäki, 1987; Taxén, 1981) that have been found to be very efficient in sampling rate conversion applications. It is also possible to design LWD filters to have an approximately linear phase in the passband (Jaworski \& Saramäki, 1994; Jones et al., 1991; Renfors \& Saramäki, 1986; Surma-aho, 1997; Surma-aho \& Saramäki, 1999). Such designs are suitable in applications where linear-phase finite-impulse response (FIR) filters would have an excessive signal delay, that is, in applications demanding narrow transition bandwidth. This is due to the fact that the order of linear-phase FIR filters is roughly inversely proportional to the transition bandwidth (Herrmann et al., 1973; Saramäki, 1993). In addition, those approximately linear-phase LWD filters proposed in (Surma-aho, 1997; Surma-aho \& Saramäki, 1999) are superior over their linear-phase FIR equivalents, in terms of the required number of multipliers, adders, and delay elements, in narrow-band cases, where linear-phase FIR filters have inherently a high filter order.

This section revises the transfer functions of the filter classes under consideration in this contribution. These filter classes consist of cascades of low-order LWD filters, approximately linear-phase LWD filters, and recursive Nth-band decimators and interpolators.

\subsection{Cascade Connection of LWD Filters}

When considering the parallel connection of two all-pass filters, it is well-known that the coefficient sensitivity is very low in the passband provided that the all-pass filter structures are constructed such that their transfer functions remain all-pass in spite of coefficient quantization (Regalia et al., 1988). However, the stopband sensitivity is not as good. In most cases, it has turned out that the required coefficient wordlength is roughly proportional to the required stopband attenuation (Renfors \& Saramäki, 1986). Therefore, the coefficient wordlength requirements can be reduced if the filter is realized using subfilters with lower stopband attenuations, e.g., in cascade or, more generally, as a tapped cascaded interconnection of identical subfilters (Saramäki \& Renfors, 1987).

An approach to designing recursive filters using a cascade of different LWD filters has been proposed in (Saramäki \& Yli-Kaakinen, 2002; Yli-Kaakinen, 2002; Yli-Kaakinen \& Saramäki, $1999 \mathrm{~b})$. The main advantage of this approach is that the poles of the cascaded LWD filters are further away from the unit circle compared with the direct LWD filters. This means that the number of data bits and the number of bits required for the coefficient representations can be 
significantly reduced. By properly determining the number of filter stages to be cascaded as well as their orders, all the coefficient values can be optimized to be representable as a few powers of two. This makes the proposed filter structure very attractive for VLSI implementations as under these circumstances all the coefficient values can be simply implemented using hardwired logic consisting of only shift operations as well as additions and/or subtractions, instead of using very costly general multiplier elements.

The transfer function of a cascade connection of LWD filters is given by

$$
H(z)=\prod_{k=1}^{K} H_{k}(z), \quad \text { where } \quad H_{k}(z)=\frac{1}{2}\left[A_{0}^{(k)}(z)+A_{1}^{(k)}(z)\right] .
$$

Here, the $A_{0}^{(k)}(z)^{\prime}$ s and $A_{1}^{(k)}(z)$ 's are the transfer functions of stable all-pass filters of orders $M_{0}^{(k)}$ and $M_{1}^{(k)}$, respectively. An implementation of the above transfer function is depicted in Fig. 1. In the sequel, the main emphasis is laid on synthesizing low-pass filters even though high-pass, band-pass, and band-stop filters can be designed in a similar manner as will be described in some detail in the sequel. In the low-pass case, $M_{0}^{(k)}=M_{1}^{(k)}-1$ or $M_{0}^{(k)}=$ $M_{1}^{(k)}+1$, so that $M_{0}^{(k)}+M_{1}^{(k)}$, the overall order of $H_{k}(z)$, is odd. If the $A_{0}^{(k)}(z)^{\prime}$ s and $A_{1}^{(k)}(z)^{\prime} \mathrm{s}$ are implemented as a cascade of first- and second-order wave digital all-pass structures and $M_{0}^{(k)}$ and $M_{1}^{(k)}$ are assumed to be odd and even, respectively, then the $A_{0}^{(k)}(z)^{\prime}$ s and $A_{1}^{(k)}(z)^{\prime} \mathrm{s}$ are expressible in terms of the adaptor coefficients as follows [see, e.g., (Gazsi, 1985)]:

$$
A_{0}^{(k)}(z)=\frac{-\gamma_{0}^{(k)}+z^{-1}}{1-\gamma_{0}^{(k)} z^{-1}} \prod_{\ell=1}^{L_{0}^{(k)}} \frac{-\gamma_{2 \ell-1}^{(k)}+\gamma_{2 \ell}^{(k)}\left(\gamma_{2 \ell-1}^{(k)}-1\right) z^{-1}+z^{-2}}{1+\gamma_{2 \ell}^{(k)}\left(\gamma_{2 \ell-1}^{(k)}-1\right) z^{-1}-\gamma_{2 \ell-1}^{(k)} z^{-2}} \quad \text { with } \quad L_{0}^{(k)}=\frac{M_{0}^{(k)}-1}{2}
$$

and

$$
A_{1}^{(k)}(z)=\prod_{\ell=L_{0}^{(k)}+1}^{L_{0}^{(k)}+L_{1}^{(k)}} \frac{-\gamma_{2 \ell-1}^{(k)}+\gamma_{2 \ell}^{(k)}\left(\gamma_{2 \ell-1}^{(k)}-1\right) z^{-1}+z^{-2}}{1+\gamma_{2 \ell}^{(k)}\left(\gamma_{2 \ell-1}^{(k)}-1\right) z^{-1}-\gamma_{2 \ell-1}^{(k)} z^{-2}} \quad \text { with } \quad L_{1}^{(k)}=\frac{M_{1}^{(k)}}{2}
$$

If $A_{0}^{(k)}(z)$ possesses a real pole at $z=r_{0}^{(k)}$ and $L_{0}^{(k)}$ complex-conjugate pole pairs at $z=$ $r_{\ell}^{(k)} \exp \left( \pm \mathrm{j} \theta_{\ell}^{(k)}\right)$ for $\ell=1,2, \ldots, L_{0}^{(k)}$ and $A_{1}^{(k)}(z)$ possesses $L_{1}^{(k)}$ complex-conjugate pole pairs at $z=r_{\ell}^{(k)} \exp \left( \pm \mathrm{j} \theta_{\ell}^{(k)}\right)$ for $\ell=L_{0}^{(k)}+1, L_{0}^{(k)}+2, \ldots, L_{0}^{(k)}+L_{1}^{(k)}$, then

$$
\gamma_{0}^{(k)}=r_{0}^{(k)}
$$

whereas

$$
\gamma_{2 \ell-1}^{(k)}=-\left(r_{\ell}^{(k)}\right)^{2} \quad \text { and } \quad \gamma_{2 \ell}^{(k)}=\frac{2 r_{\ell}^{(k)} \cos \left(\theta_{\ell}^{(k)}\right)}{1+\left(r_{\ell}^{(k)}\right)^{2}} \quad \text { for } \ell=1,2, \ldots, L_{0}^{(k)}+L_{1}^{(k)}
$$




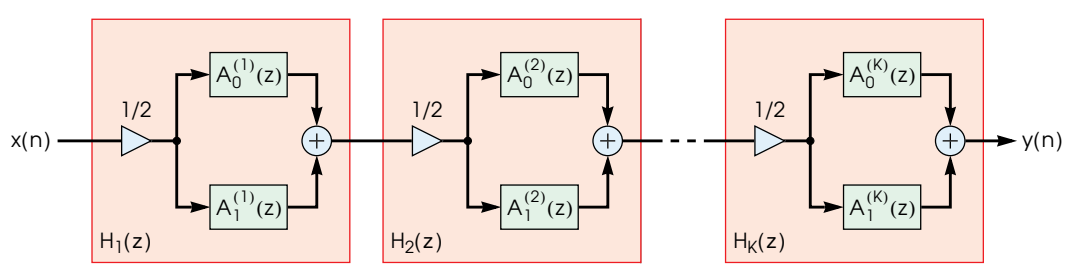

Fig. 1. Filter structure for a cascade connection of LWD filters. The detailed implementation of the $k$ th transfer function $H_{k}(z)$ as a parallel connection of $A_{0}^{(k)}(z)$ and the $A_{1}^{(k)}(z)$ is shown in Fig. 2.

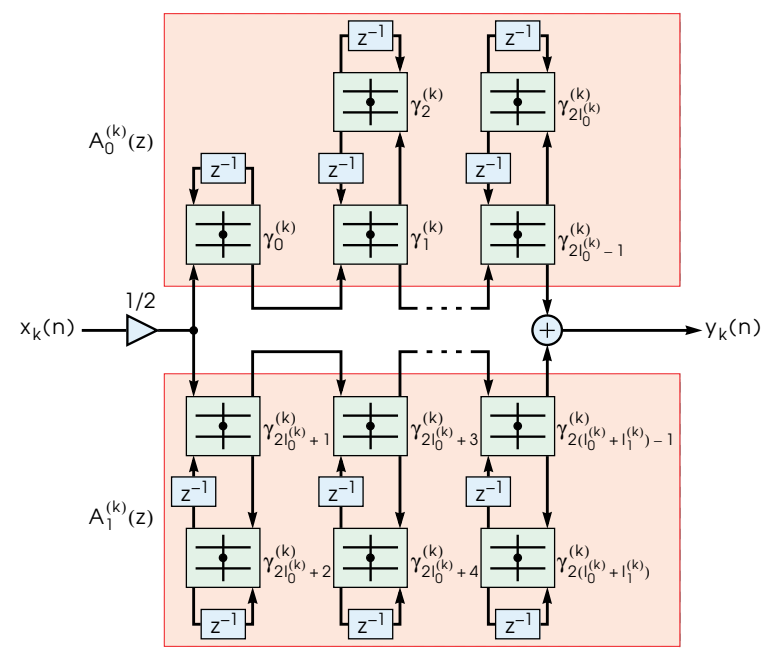

Fig. 2. Implementation of the $k$ th transfer function in Fig. 1 as a parallel connection of two all-pass filter transfer functions. $A_{0}^{(k)}(z)$ and $A_{1}^{(k)}(z)$ are stable all-pass filter transfer functions consisting of a cascade of first- and second-order wave digital all-pass sections. These firstand second-order wave digital all-pass sections are constructed based on the use of two-port adaptor structures to be described in Section 3.

Figure 2 shows the realization for a low-pass sub-filter transfer function $H_{k}(z)$, where the firstand second-order sections of $(2 a)$ and $(2 b)$ are implemented as a cascade of first- and secondorder wave-digital all-pass structures, out of which the best ones for the main purposes of this book chapter will be considered in detail in Section 3.

In the high-pass case, the corresponding transfer function is obtained by simply changing the sign of $A_{0}^{(k)}(z)$ or $A_{1}^{(k)}(z)$ in (1) (Gazsi, 1985). In the band-stop case, $M_{0}^{(k)}$ and $M_{1}^{(k)}$ are two times an odd integer and an even integer, respectively, and $M_{0}^{(k)}=M_{1}^{(k)}-2$ or $M_{0}^{(k)}=M_{1}^{(k)}+$ 2. The corresponding band-pass design can be generated by changing the sign of $A_{0}^{(k)}(z)$ or $A_{1}^{(k)}(z)$. The main difference of the band-pass and band-stop filter designs in comparison with the low-pass and high-pass filter designs is thus that the first-order section is absent. 


\subsection{Approximately Linear-Phase LWD Filters}

One of the most difficult problems in digital filter synthesis is the simultaneous optimization of the phase and magnitude responses of recursive digital filters. This is because the phase of recursive filters is inherently nonlinear and, therefore, the frequency selectivity and phase linearity are conflicting requirements. The most straightforward approach to arrive at a recursive filter having simultaneously a selective magnitude response and an approximately linear-phase response in the passband region is to generate the filter in two steps. First, a filter with the desired magnitude response is designed. Then, the phase response of this filter is made approximately linear in the passband by cascading it with an all-pass phase equalizer (Deczky, 1972; Rabiner \& Gold, 1975). The main drawback in this approach is that the phase response of the frequency-selective filter is usually very nonlinear and, therefore, a very high-order phase equalizer is needed in order to make the phase response of the overall filter approximately linear.

It has turned out (Földvári-Orosz et al., 1991; Jaworski \& Saramäki, 1994; Jones et al., 1991; Lawson \& Wicks, 1992; Leeb, 1991; Surma-aho, 1997; Surma-aho \& Saramäki, 1999) to be more beneficial to implement an approximately linear-phase recursive filter directly without using a separate phase equalizer. In the design techniques described in (Földvári-Orosz et al., 1991; Jaworski \& Saramäki, 1994; Jones et al., 1991; Lawson \& Wicks, 1992; Leeb, 1991; Surmaaho, 1997; Surma-aho \& Saramäki, 1999), it has been observed that in order to simultaneously achieve a selective magnitude response and an approximately linear-phase performance in the passband, it is required that some zeros of the filter be located outside the unit circle.

For approximately linear-phase LWD filters, it has been discovered in (Saramäki \& YliKaakinen, 2002) that the use of a cascade of several filter blocks does not provide any benefits in the VLSI implementations. Therefore, the transfer function for the approximately linearphase LWD filters is given by (1) with $K=1$, that is, $H(z)$ is expressible as

$$
H(z)=\frac{1}{2}\left[A_{0}^{(1)}(z)+A_{1}^{(1)}(z)\right],
$$

where $A_{0}^{(1)}(z)$ and $A_{1}^{(1)}(z)$ are given by (2a) and (2b), respectively.

\subsection{Recursive $N$ th-Band Decimators and Interpolators}

The best structures for implementing decimation and interpolation filters in cases where the phase linearity is not important, are the so-called recursive Nth-band filters (Renfors \& Saramäki, 1987; Saramäki \& Renfors, 1998; Yli-Kaakinen et al., 1999). ${ }^{1}$ These recursive Nthband filters when used alone for decimation by the factor of $N$ suffer, due to their properties, from the drawback that, after specifying the passband edge to be $\omega_{p}=\alpha \pi / N$ with $\alpha<1$, only aliasing into the passband region $\left[0, \omega_{p}\right]$ can be fully avoided, but aliasing into the transition band $\left[\omega_{p}, \pi / N\right]$ occurs. In the interpolation case, this causes the corresponding imaging effects. If these effects can be tolerated and a linear-phase performance is not required, then these recursive polyphase filters require the lowest computational complexities among the known decimators and interpolators. From a computational point of view, it is very advantageous to use multistage decimators and interpolators whenever possible, instead of using a single-stage realization. The design of recursive $N$ th-band filters and their use for decimation

\footnotetext{
${ }^{1}$ It is also possible to design recursive $N$ th-band filters to have an approximately linear-phase response in the passband (Ansari \& Liu, 1983; Renfors \& Saramäki, 1987). These filters require significantly higher computational complexities than the corresponding nonlinear-phase $N$ th-band filters, but they compare favorably with conventional linear-phase FIR filters.
} 


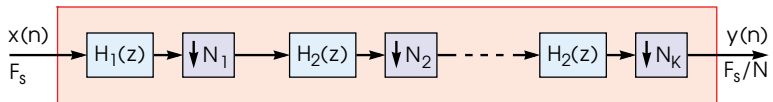

(a)

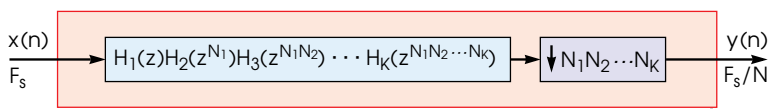

(b)

Fig. 3. (a) A general implementation form for an $N$-to-1 decimator. (b) Its single-stage equivalent.

and interpolation has been discussed in detail in (Renfors \& Saramäki, 1987). In this article, it has also been described how to get around the above-mentioned drawbacks by using an additional LWD filter at the output of the overall decimator or at the input of the overall interpolator.

Due to the duality between decimators and interpolators, the discussion in this book chapter will concentrate on the design of decimators. If the sampling rate conversion ratio can be factored into the product

$$
N=\prod_{k=1}^{K} N_{k}
$$

where $N_{1}, N_{2}, \ldots, N_{K}$ are integers, then the overall decimation by the factor of $N$ can be implemented using $K$ stages as shown in Fig. 3(a) (Renfors \& Saramäki, 1987). In order to considerably clarify the analysis and determination of the roles of the sub-blocks of Fig. 3(a) in simultaneously providing the desired decimation by the overall factor of $N$, it is advantageous to replace the implementation of Fig. 3(a) by its its single-stage equivalent of Fig. 3(b). In this equivalent, only one filter with transfer function

$$
H(z)=\prod_{k=1}^{K} H_{k}\left(z^{\widetilde{N}_{k}}\right), \quad \text { where } \quad \widetilde{N}_{1}=1 \quad \text { and } \quad \widetilde{N}_{r}=\prod_{k=1}^{r-1} N_{k} \quad \text { for } r=2,3, \ldots, K
$$

is involved followed by decimation by a factor of $N$. The magnitude response of the above overall filter is thus

$$
\left|H\left(\mathrm{e}^{\mathrm{j} \omega}\right)\right|=\prod_{k=1}^{K} H_{k}\left(\mathrm{e}^{\mathrm{j} \widetilde{N}_{k} \omega}\right) .
$$

When the transfer functions $H_{k}(z)$ for $k=1,2, \ldots, K$ in Fig. 3(a) are implemented with the aid of the $K$ recursive (nonlinear-phase) $N_{k}$ th-band filters, where $N_{k}$ is the decimation factor after the $k$ th subfilter, the transfer function in the single-stage equivalent of Fig. $3(\mathrm{~b})$ is used as a basic transfer function when synthesizing Nth-band decimators. For this purpose, this transfer function is expressed as

$$
H(z)=\prod_{k=1}^{K} H_{k}\left(z^{\widetilde{N}_{k}}\right), \quad \text { where } \quad H_{k}(z)=\frac{1}{N_{k}} \sum_{n=0}^{N_{k}-1} z^{-n} A_{n}^{(k)}\left(z^{N_{k}}\right) .
$$




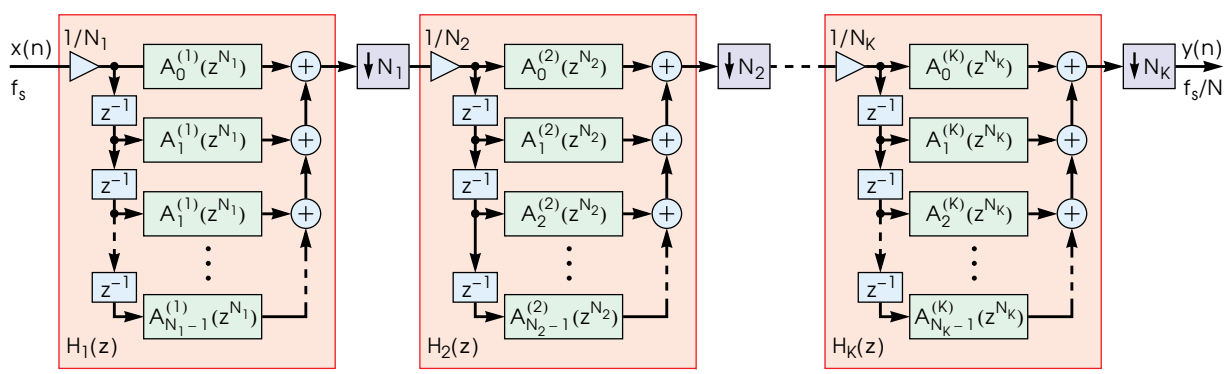

Fig. 4. Filter structure for multistage recursive Nth-band decimators. The $A_{n}^{(k)}(z)$ 's are the transfer functions of stable all-pass filters consisting of a cascade of first-order wave-digital all-pass sections.

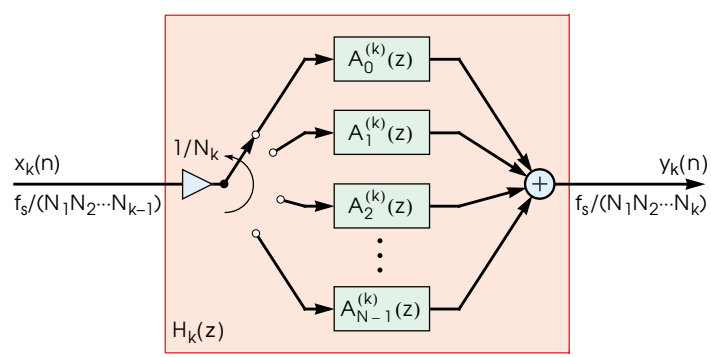

Fig. 5. Commutative structure for the $k$ th stage in Fig. 4.

Here, the transfer functions $A_{n}^{(k)}(z)$ are the following cascades of first-order stable all-pass transfer functions:

$$
A_{n}^{(k)}(z)=\prod_{\ell=\widetilde{L}_{n}^{(k)}+1}^{\widetilde{L}_{n}^{(k)}+L_{n}^{(k)}} \frac{-\gamma_{\ell}^{(k)}+z^{-1}}{1-\gamma_{\ell}^{(k)} z^{-1}} \text { for } n=0,1, \ldots, N_{k}-1 \text { and for } k=1,2, \ldots, K,
$$

where

$$
\widetilde{L}_{0}=0 \quad \text { and } \quad \widetilde{L}_{n}^{(k)}=\sum_{r=0}^{n-1} L_{r}^{(k)} \text { for } n=0,1, \ldots, N_{k}-1 .
$$

Hence, each $A_{n}^{(k)}$ for $n=0,1, \ldots, N_{k}-1$ and for $k=1,2, \ldots, K$ possesses $L_{n}^{(k)}$ real poles at $z=r_{\ell}^{(k)}=\gamma_{\ell}^{(k)}$ for $\ell=\widetilde{L}_{n}^{(k)}+1, \widetilde{L}_{n}^{(k)}+2, \ldots, \widetilde{L}_{n}^{(k)}+L_{n}^{(k)}$.

The transfer function of (8a), (8b), and (8c) corresponds to the decimation structure of Fig. 4 . From the practical implementation point of view, this structure becomes very attractive if the $k$ th transfer function followed by decimation by the factor of $N_{k}$ is replaced by the highly efficient commutative structure of Fig. 5 (Crochiere \& Rabiner, 1983). The advantages of this structure are that the delay line is not needed and the branch filters $A_{n}^{(k)}\left(z^{N_{k}}\right)$ 's are implemented as $A_{n}^{(k)}(z)$ 's at the lower sampling rate. This reduces by the factor of $N_{k}$ both the number of multiplications per input sample and the delay terms required for implementing the branch filters. 


\section{Coefficient Representation under Consideration}

This contribution concentrates on the coefficient quantization in fixed-point arithmetic. In many implementations, it is attractive to carry out the multiplication of a data sample by a filter coefficient value using a sequence of shifts and adds and/or substracts. For such a purpose, it is desirable to express the coefficient values in the form

$$
\sum_{r=1}^{R} a_{r} 2^{-P_{r}},
$$

where each of the $a_{r}$ 's is either 1 or -1 and the $P_{r}$ 's are non-negative integers in the increasing order.

The goal in optimization problems stated in Section 4 is to minimize the implementation cost by finding all the coefficient values in such a way that, first, $R$, the number of powers of two, is made as small as possible and, then, $P_{R}$, the number of fractional bits, is made as small as possible.

A reasonable estimate for the implementation cost of the filter is the number of adders and/or subtracters required to implement all the adaptor coefficients. When using this estimate, the overall silicon area and the power consumption required by the full-custom VLSI implementation of the filter is roughly minimized (Ohlsson et al., 2001; Wanhammar, 1998).

It should be pointed out that, in addition to adders and/or subtracters needed for the adaptor coefficients, several structural adders are also required for implementing the wave-digital allpass sections. These first- and second-order wave-digital all-pass sections are constructed based on the use of two-port adaptor structures and delays as depicted in Fig 2. For LWD filters, there exists a great variety of adaptor structures according to the realization possibilities of the analog reference filters (Fettweis, 1986; Fettweis et al., 1974; Gazsi, 1985). The actual multipliers to be implemented and the number of structural adders required to implement the two-port adaptor structures depends on the selected adaptor type.

Figure 6 shows particular symmetric two-port adaptor structures that lead to the optimal scaling for a sinusoidal excitation according to the discussion in (Gazsi, 1985). However, it has been shown, based a further study performed in (Renfors \& Zigouris, 1988), that in some cases for the second-order wave-digital all-pass sections, the additional scaling factors $c$ and $1 / c$ are required at the input and the output of the second adaptor, respectively, in order to achieve the optimal scaling. In order to keep the resulting second-order sections still all-pass, $c$ must be a (positive or negative) power of two. Due to this fact, the above improved scaling has no effect on the overall procedure and the results achieved in this contribution.

The selection among the four optional structures of Fig. 6 depends on the value of the multiplier $\gamma$ such that the structures of Figs. 6(a), 6(b), 6(c), and 6(d) are chosen for $\frac{1}{2}<\gamma<1$, $0<\gamma \leq \frac{1}{2},-\frac{1}{2} \leq \gamma<0$, and $-1<\gamma<-\frac{1}{2}$, respectively. In these cases, the value of $\alpha$, the actual multiplier to be implemented, depends on the value of $\gamma$ as follows:

$$
\alpha= \begin{cases}1-\gamma & \text { for } \quad \frac{1}{2}<\gamma<1 \\ \gamma & \text { for } 0<\gamma \leq \frac{1}{2} \\ -\gamma & \text { for }-\frac{1}{2} \leq \gamma<0 \\ 1+\gamma & \text { for }-1<\gamma<-\frac{1}{2}\end{cases}
$$

Consequently, the value of $\alpha$ is always positive and less than or equal to half. Therefore, when the absolute value of $\gamma$ is greater than half, the number of adders required for implementing the corresponding $\alpha$ coefficient decreases by one. 


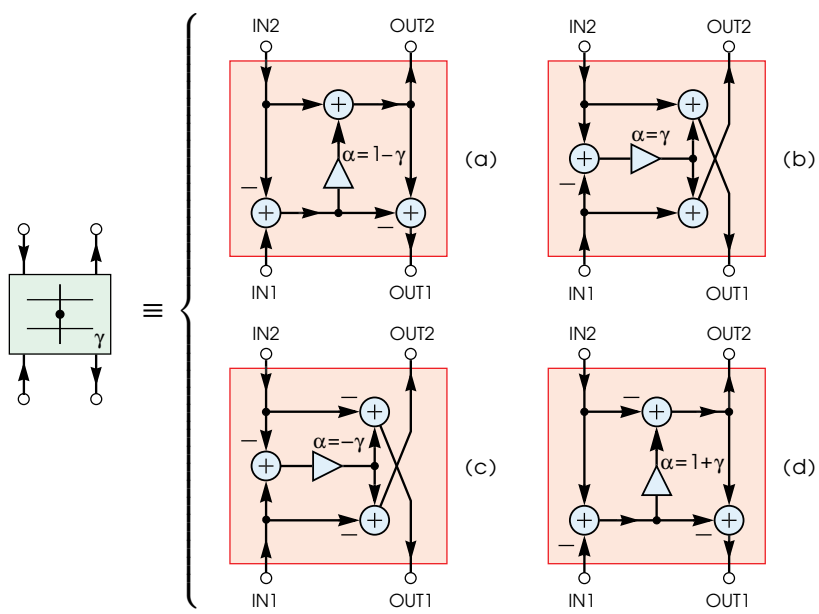

Fig. 6. Efficient two-port adaptor structures yielding optimal scaling for a sinusoidal excitation (Gazsi, 1985).

\section{Optimization Problems for the Filter Classes under Consideration}

This chapter summarizes the optimization problems for all the three filter classes under consideration in this book chapter. For each filter class, the specifications, the adjustable parameter vector, and the optimization problem will be described.

Before stating the optimization problem for each of the above-mentioned three filter classes, the transfer function for each filter class is denoted in the same manner by $H(\Phi, z)$, where $\Phi$ is the adjustable parameter vector containing the adjustable parameters which depend on the filter class at hand in a manner to be described later on. Similarly, the magnitude criteria are stated in the common manner as follows. Given $\Omega_{p}$ and $\Omega_{s}$, the passband and stopband regions, respectively, as well as $\delta_{p}$ and $\delta_{s}$, the passband and stopband ripples, respectively, the magnitude specifications for the filter are stated as follows:

$$
\begin{aligned}
1-\delta_{p} \leq\left|H\left(\Phi, \mathrm{e}^{\mathrm{j} \omega}\right)\right| \leq 1 \quad \text { for } \omega \in \Omega_{p} \\
\left|H\left(\Phi, \mathrm{e}^{\mathrm{j} \omega}\right)\right| \leq \delta_{s} \quad \text { for } \omega \in \Omega_{s} .
\end{aligned}
$$

It is worth pointing out that these specifications are typical of most recursive filters built using all-pass filters as building blocks as, in these most cases, the filter structure constrains the maximum of the magnitude response to be unity. Alternatively, the above criteria are expressible as

$$
\begin{array}{cl}
|E(\Phi, \omega)| \leq 1 \quad \text { for } \omega \in \Omega_{p} \cup \Omega_{S} \\
E(\Phi, \omega) \leq 0 \quad \text { for } \omega \in \Omega_{p},
\end{array}
$$

where

$$
E(\Phi, \omega)=W(\omega)\left[\left|H\left(\Phi, \mathrm{e}^{\mathrm{j} \omega}\right)\right|-D(\omega)\right]
$$

with

$$
D(\omega)=\left\{\begin{array}{ll}
1 & \text { for } \omega \in \Omega_{p} \\
0 & \text { for } \omega \in \Omega_{s}
\end{array} \text { and } W(\omega)= \begin{cases}1 / \delta_{p} & \text { for } \omega \in \Omega_{p} \\
1 / \delta_{s} & \text { for } \omega \in \Omega_{s} .\end{cases}\right.
$$


As the third option for later use, the above magnitude criteria are stated as

$$
\begin{array}{r}
0 \leq 20 \log _{10}\left|H\left(\Phi, \mathrm{e}^{\mathrm{j} \omega}\right)\right| \leq-A_{p} \quad \text { for } \omega \in \Omega_{p} \\
20 \log _{10}\left|H\left(\Phi, \mathrm{e}^{\mathrm{j} \omega}\right)\right| \leq-A_{s} \quad \text { for } \omega \in \Omega_{s},
\end{array}
$$

where

$$
A_{p}=-20 \log _{10}\left(1-\delta_{p}\right) \text { and } A_{s}=-20 \log _{10}\left(\delta_{s}\right)
$$

are the admissible positive passband variation and stopband attenuation, respectively. These criteria will be mainly used in connection of Examples of Section 6 for specifying the magnitude criteria for the three filter classes under consideration.

The target in all of the following optimization problems is to find the quantized values of the adaptor coefficients corresponding to the parameter values included in $\Phi$ such that, first, the coefficient values are expressible in the form of (9) and, second, the number of adders and subtracters required to implement all the adaptor coefficient is minimized.

\subsection{Cascade Connection of LWD Filters}

According to the construction of the overall transfer function for these filters in Subsection 2.1 by means of $(1),(2 a),(2 b),(3 a)$, and (3b), the optimization problem is stated in the low-pass case as follows: Find $K$, the number of sub-stages, $M_{0}^{(k)}$ and $M_{1}^{(k)}$ for $k=1,2, \ldots, K$, the orders of the all-pass subfilters, as well as the adjustable parameter vector as given by

$$
\begin{aligned}
\Phi= & {\left[r_{0}^{(1)}, r_{1}^{(1)}, \ldots, r_{L_{0}^{(1)}+L_{1}^{(1)}}^{(1)}, \theta_{1}^{(1)}, \theta_{2}^{(1)}, \ldots, \theta_{L_{0}^{(1)}+L_{1}^{(1)}}^{(1)}\right.} \\
& r_{0}^{(2)}, r_{1}^{(2)}, \ldots, r_{L_{0}^{(2)}+L_{1}^{(2)}}^{(2)}, \theta_{1}^{(2)}, \theta_{2}^{(2)}, \ldots, \theta_{L_{0}^{(2)}+L_{1}^{(2)}}^{(2)} \ldots, \\
& \left.r_{0}^{(K)}, r_{1}^{(K)}, \ldots, r_{L_{0}^{(K)}+L_{1}^{(K)}}^{(K)}, \theta_{1}^{(K)}, \theta_{2}^{(K)}, \ldots, \theta_{L_{0}^{(K)}+L_{1}^{(K)}}^{(K)}\right],
\end{aligned}
$$

in such a way that the criteria given by (12a)-(12d) are met and the above-mentioned target for the coefficient implementations is achieved.

\subsection{Approximately Linear-Phase LWD Filters}

In the sequel, when synthesizing approximately linear-phase low-pass LWD filters, in addition to the magnitude criteria of $(12 \mathrm{a})-(12 \mathrm{~d})$, the phase requirements are stated as follows (Surma-aho \& Saramäki, 1999):

$$
\left|\arg H\left(\Phi, \mathrm{e}^{\mathrm{j} \omega}\right)-\tau \omega\right| \leq \Delta \text { for } \omega \in \Omega_{p} .
$$

Here, $\arg H\left(\Phi, \mathrm{e}^{\mathrm{j} \omega}\right)$ denotes the unwrapped phase response of the filter, whereas $\tau$ is the value minimizing the maximum absolute value of $\arg H\left(\Phi, \mathrm{e}^{\mathrm{j} \omega}\right)-\tau \omega$ on the passband region $\Omega_{p}$ and $\Delta$ is the upper limit for this maximum. Since only a single LWD filter is under optimization, the adjustable vector reduces to

$$
\Phi=\left[r_{0}^{(1)}, r_{1}^{(1)}, \ldots, r_{L_{0}^{(1)}+L_{1}^{(1)}}^{(1)} \theta_{1}^{(1)}, \theta_{2}^{(1)}, \ldots, \theta_{L_{0}^{(1)}+L_{1}^{(1)}}^{(1)}\right] .
$$

In this case, the optimization problem is the following: Find $M_{0}^{(1)}$ and $M_{1}^{(1)}$, the orders of the all-pass subfilters, as well as the adjustable parameter vector $\Phi$, as given by (16), in such a 
way that in addition to meeting the magnitude criteria of (12a)-(12d), the phase specifications of (15) are satisfied and the above-mentioned target for the coefficient implementations is achieved.

\subsection{Recursive $N$ th-Band Decimators and Interpolators}

If the desired sampling rate conversion factor is $N$, then the passband region of the decimation filter is selected as $\Omega_{p}=\left[0, \omega_{p}\right]$ where $\omega_{p}<\pi / N$. The selection of the stopband region $\Omega_{s}$ depends on whether or not aliasing is allowed into the transition band $\left[\omega_{p}, \pi / N\right]$ of the filter. Due to the properties of recursive Nth-band filters, their stopband region for the abovespecified passband region is inherently restricted to be (Renfors \& Saramäki, 1987)

$$
\Omega_{S}=\bigcup_{r=1}^{\lfloor N / 2\rfloor}\left[r \frac{2 \pi}{N}-\omega_{p}, \min \left(r \frac{2 \pi}{N}+\omega_{p}, \pi\right)\right] .
$$

This region has the following properties. First, for $N>3, \Omega_{s}$ is a multiband stopband region that consist of $\lfloor N / 2\rfloor$ bands such that the first $\lfloor N / 2\rfloor-1$ bands are $\left[r 2 \pi / N-\omega_{p}, r 2 \pi / N+\right.$ $\left.\omega_{p}\right]$ for $r=1,2, \ldots,\lfloor N / 2\rfloor-1$ and the last band is $\left[\pi-\omega_{p}, \pi\right]$ and $\left[(N-1) 2 \pi / N-\omega_{p},(N-\right.$ 1) $\pi / N+\omega_{p}$ ] for $N$ even and odd, respectively (As a typical example, see Fig. 19 in Subsection 6.3 showing the magnitude response for a finite-precision eighth-band $(N=8)$ design.). Second, for $N=2$ and $N=3, \Omega_{s}=\left[\pi-\omega_{p}, \pi\right]$ and $\Omega_{s}=\left[2 \pi / 3-\omega_{p}, 2 \pi / 3+\omega_{p}\right]$, respectively. Therefore, first, the lower edge of the first stopband region is located at $\omega=2 \pi / N-\omega_{p}$ and, second, $\Omega_{s}$ has for $N>2$, in addition to the transition band of width $2\left(\pi / N-\omega_{p}\right)$, don't care bands of the same width around $\omega_{r}=(2 r+1) \pi / N$ for $r=1,2, \ldots,\lfloor(N+1) / 2\rfloor-1$. The above stopband region guarantees that the aliasing is fully avoidable into the passband region. If this control is desired to extend onto $[0, \pi / N]$, then an additional LWD filter can be implemented after the overall decimation (Renfors \& Saramäki, 1987).

This book chapter concentrates on the design of those single-stage and multistage recursive Nth-band decimators, where this additional LWD filter is excluded. For this purpose, the following second main characteristics of the recursive $N$ th-band filters is utilized. If the maximum magnitude value of the filter on $\Omega_{S}$ is $\delta_{S}$, then it is guaranteed that in the minimum magnitude value on the passband region $\left[0, \omega_{p}\right]$ is larger than or equal $\sqrt{1-(N-1)\left(\delta_{S}\right)^{2}}$ (Renfors \& Saramäki, 1987). This implies that for any practical stopband attenuation on $\Omega_{S}$, the passband variation becomes negligible. Consequently, the design of recursive Nth-band decimator can concentrate on the stopband region $\Omega_{S}$ only. Therefore, the criteria of (11) can be reduced into the following form:

$$
E(\Phi, \omega)=\left|H\left(\Phi, e^{\mathrm{j} \omega}\right)\right| \leq \delta_{s} \quad \text { for } \omega \in \Omega_{s},
$$

where $\Omega_{S}$ is given by (17).

According to the construction of the overall transfer function in the single-stage equivalent in Subsection 2.3 by means of (5), (6), (8a), (8b), and (8c), the optimization problem is stated as follows: Find $K$, the number of sub-stages, $N_{1}, N_{2}, \ldots, N_{K}$, the decimation factors of the substages, the $L_{n}^{(k)}$ 's, the orders of the branch filters, as well as the adjustable parameter vector as 
given by

$$
\begin{aligned}
\Phi= & {\left[r_{1}^{(1)}, r_{2}^{(1)}, \ldots, r_{L_{0}^{(1)}+L_{1}^{(1)}+\cdots+L_{N_{1}-1}^{(1)}}^{(1)},\right.} \\
& r_{1}^{(2)}, r_{2}^{(2)}, \ldots, r_{L_{0}^{(2)}+L_{1}^{(2)}+\cdots+L_{N_{2}-1}^{(2)}, \ldots,}^{(2)}, \ldots, \\
& \left.r_{1}^{(K)}, r_{2}^{(K)}, \ldots, r_{L_{0}^{(K)}+L_{1}^{(K)}+\cdots+L_{N_{K}-1}^{(K)}}^{(K)}\right],
\end{aligned}
$$

in such a way that criteria given by (18) are met and the above-mentioned target for the coefficient implementations is achieved.

\section{Filter Optimization}

The solutions to the three optimization problems stated in the previous section can be found in a similar manner by using the following three steps. In the first step, a filter with infiniteprecision coefficients is determined in such a way that it exceeds the given frequency-domain criteria in order to provide some tolerance for coefficient quantization. Then, in the second step, the smallest and largest values are determined for each adjustable parameter by reoptimizing the remaining unknowns in the parameter vector in such a manner that the given specifications are met. This enables one to find the parameter space of the infinite-precision coefficients including the feasible space where the filter meets the specifications. Finally, the third step involves finding the filter parameters in this space so that the resulting filter meets the given criteria with the simplest coefficient representation forms. This strategy is general but particularly efficient for LWD filters due to the fact that for these filters only the denominator coefficients of the all-pass sections have to be quantized.

The proposed quantization scheme provides significant advantages over those based on the use of other existing techniques. First of all, it is always guaranteed that the optimum solution can be found to the above three optimization problems. Second, the computational workload to arrive at the optimum finite-precision solution is in most cases significantly smaller than in other existing techniques.

\subsection{Generating the Initial Infinite-Precision Solution}

In many cases, finding a good initial solution is not trivial as it implies a good understanding and characterization of the problem. Furthermore, for each problem at hand the way of generating the start-up solution is very different. If there is a systematic approach for finding an initial solution being close to the optimum one, then the above-described three-step procedure gives in most cases more quickly a solution that is better than those obtained, e.g., by using simulated annealing or genetic algorithms.

\subsubsection{Cascade connection of LWD filters}

The design of an initial conventional LWD filter for further optimization can be carried out by, first, using an appropriate classical analog-filter approximation and, then, converting the resulting continuous-time transfer function into a desired discrete-time transfer function (Antoniou, 1993; Rabiner \& Gold, 1975; Schüßler, 2010). Another approach for designing an initial filter is to use explicit formulas developed directly for digital filters in (Gazsi, 1985). It is well known that the odd-order elliptic filter is the most selective low-pass or high-pass filter being implementable as a parallel connection of two all-pass filters [see, e.g., (Gazsi, 1985)]. For 


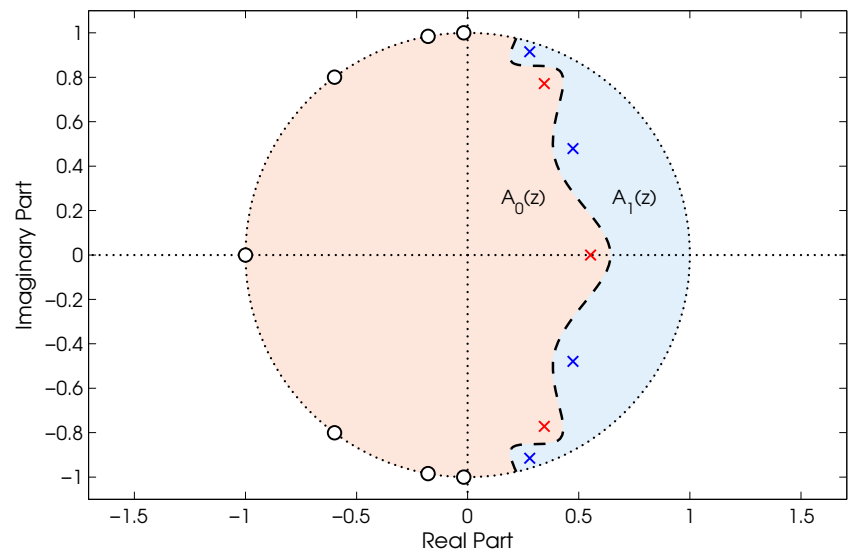

Fig. 7. Alternating distribution for the poles of a prototype filter among the two all-pass filters $A_{0}(z)$ and $A_{1}(z)$ for a seventh-order low-pass filter with $\omega_{p}=0.4 \pi, \omega_{s}=0.5 \pi, A_{p}=0.2 \mathrm{~dB}$ $\left(\delta_{p}=0.0228\right)$, and $A_{s}=60 \mathrm{~dB}\left(\delta_{s}=10^{-3}\right)$.

conventional low-pass, high-pass, band-pass and, band-stop criteria, the order of an elliptic filter meeting the given specifications can be estimated using the well-known approximation formulas (Antoniou, 1993; Rabiner \& Gold, 1975; Schüßler, 2010).

Since the real pole and the complex-conjugate pole pairs of the all-pass filters for low-pass and high-pass designs have the real zero and complex-conjugate zero pairs in conjugate reciprocal positions (Antoniou, 1993; Schüßler, 2010), the poles of the designed filter unambiguously determine the all-pass filters. After knowing the poles of the filter, the problem is to implement the overall transfer function in such a way that the poles are properly shared between the two all-pass sections $A_{0}(z)$ and $A_{1}(z)$. If the poles are distributed in the low-pass case in a regular manner, then $A_{0}(z)$ can be selected to realize the real pole, the second innermost complexconjugate pole pair, the fourth innermost complex-conjugate pole pair and so on, whereas $A_{1}(z)$ realizes the remaining poles (Gazsi, 1985). For a very complicated pole distribution, the procedure described in (Saramäki, 1985) can be used for sharing the poles between $A_{0}(z)$ and $A_{1}(z)$. The alternating distribution of the poles among the two all-pass filters for a seventhorder elliptic prototype filter is illustrated in Fig. 7.

The above discussion applies directly to a single LWD filter. For the cascades of low-order LWD filters, in turn, it has turned out to be advantageous in most cases to select all the $A_{0}^{(k)}(z)^{\prime}$ 's and the $A_{1}^{(k)}(z)^{\prime}$ s to be of the same order, respectively. In this case, the starting point filter for further optimization can be determined by using several identical copies of the same subfilter. For $K$ identical copies of the same subfilter, the passband and stopband ripples for this subfilter should be approximately equal to $\delta_{p} / K$ and $\sqrt[K]{\delta_{s}}$, respectively. There is clearly a trade-off between $K$, the number of subfilters, and the order of the subfilter; the higher is the value of $K$, the lower is the order of the subfilter. However, since the subfilter order is restricted to be an odd integer, there are only a few practical combinations for the subfilter order and $K$. It is not necessary for the subfilter being an odd-order elliptic filter to exactly meet the ripple requirements. This is due to the fact that further optimization makes the subfilters different and simultaneously improves the overall filter performance. 


\subsubsection{Approximately linear-phase LWD filters}

For these low-pass LWD filters, there exist no closed-form solution for satisfying both the magnitude criteria of (12a)-(12d) and the phase criteria of (15). Therefore, these filters have to be designed using optimization techniques. An efficient systematic algorithm for designing an initial solution for these filters has been proposed in (Surma-aho, 1997; Surma-aho \& Saramäki, 1999). This design scheme consists of two basic steps. The first step involves finding in a simple straightforward manner a good suboptimal solution that determines $\Phi$ so that $\Delta$ in (15) has a reasonably small value subject to the magnitude specifications. In the second step, this solution is then used as an initial filter for further optimization carried out with the aid of a constrained optimization for minimizing the value of $\Delta$ in (15) subject to the magnitude criteria.

\subsubsection{Recursive $N$ th-band decimators and interpolators}

The initial infinite-precision solutions for the recursive Nth-band filter in both the singlestage and multistage implementations can be properly synthesized by utilizing the synthesis schemes described in (Renfors \& Saramäki, 1987). The design of single-stage filters relies on the properties of these filters and enables one to significantly reduce the number of the original unknowns. Furthermore, the remaining unknowns can be found by means of an efficient Remez-type algorithm. As a result, solutions being very close to the optimized solutions can be achieved in a very fast and reliable manner in comparison with other existing very timeconsuming optimization techniques, which are based on optimizing the original unknowns and do not necessarily guarantee the arrival at the optimized solution.

The multistage design, in turn, counts on the fact that each stage, as has been observed in (Renfors \& Saramäki, 1987), has its own predetermined frequency range to take care of in order to provide the desired magnitude response for the overall design. Based on this fact, the simultaneous design of the sub-stages can be conveniently performed by iteratively determining them such that they provide for the overall filter as high attenuation as possible in their predetermined frequency ranges. This iteration is continued until the successive overall solutions become practically the same. What is left is to determine the minimum filter orders to meet the given specifications.

\subsection{Optimization of Infinite-Precision Filters}

The optimization algorithm is based on the following observation. Finding the smallest and largest values for each adjustable parameter by reoptimizing the remaining unknowns in the parameter vector so that the given criteria are still met enables one to determine a parameter space including the feasible space where the filter specifications are satisfied. After figuring out this space, all that is needed is to check whether in this space there exist the desired discrete values for the given coefficient representation form.

\subsubsection{Cascade connection of LWD filters}

For cascaded LWD filters, the parameter space of the infinite-precision coefficients can be determined as follows. For each complex-conjugate pole pair, the smallest and largest values for both the radius and the angle are determined so that by reoptimizing the locations of the remaining poles the given overall magnitude criteria of (12a)-(12d) can still be met. For the real pole, the smallest and largest values for the radius are found in the same manner.

The above procedure gives for the upper-half-plane pole of each complex-conjugate pole pair $r_{\ell}^{(k)} \exp \left( \pm \mathrm{j} \theta_{\ell}^{(k)}\right)$ for $\ell=1,2, \ldots, L_{0}^{(k)}+L_{1}^{(k)}$ and for $k=1,2, \ldots, K$, the region $R \exp (\mathrm{j} \Theta)$ where 


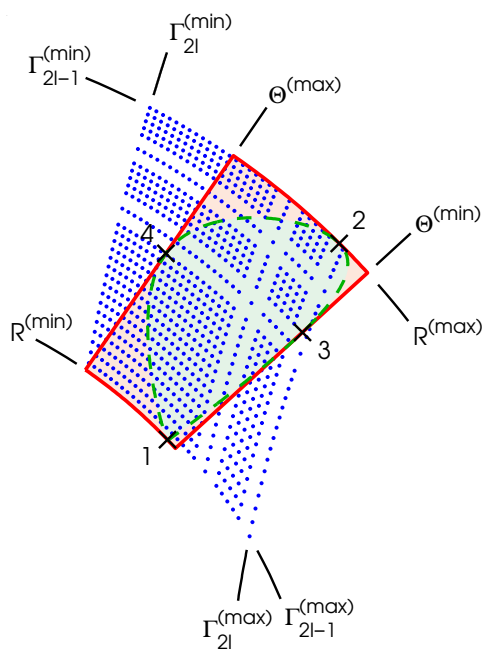

(a)

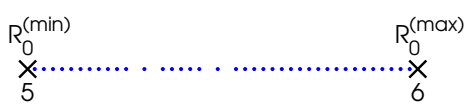

(b)

Fig. 8. Typical search spaces for the poles when three powers of two with seven fractional bits $\left(R=3\right.$ and $\left.P_{R}=7\right)$ are used for the adaptor coefficients. (a) Upper-half-plane pole for the complex-conjugate pole pair. (b) Real pole.

$R^{(\min )} \leq R \leq R^{(\max )}$ and $\Theta^{(\min )} \leq \Theta \leq \Theta^{(\max )}$, as illustrated in Fig. 8(a). The crosses numbered by $1,2,3$, and 4 correspond, respectively, to the points where the smallest radius $R^{(\min )}$, the largest radius $R^{(\max )}$, the smallest angle $\Theta^{(\mathrm{min})}$, and the largest angle $\Theta^{(\max )}$ are reached. Inside this region, there is the feasible region, given by the dashed line in Fig. 8(a), where the pole can be located such that by relocating the remaining poles the given overall criteria are still met by using an infinite-precision arithmetic. For each real pole $r_{0}^{(k)}$ for $k=$ $1,2, \ldots, K$, there exists the corresponding region $R_{0}^{(\min )} \leq R \leq R_{0}^{(\max )}$ that is simultaneously the feasible region. In Fig. 8(b), the crosses numbered by 5 and 6 indicate $R_{0}^{(\min )}$ and $R_{0}^{(\max )}$, respectively.

For the complex-conjugate pole pairs, the larger region is used because it can be found very quickly by applying only four times the algorithm to be described next. For the real pole, there is a need to use this algorithm only twice. Hence, in order to find the above-mentioned regions for all the poles of the low-pass transfer function, as given by (1), (2a), (2b), (3a), and (3b), there are for each of the $K$ sub-stages $2+4\left(L_{0}^{(k)}+L_{1}^{(k)}\right)$ problems of the following form: Find the adjustable parameter vector $\Phi$ to minimize $\psi$ subject to the conditions of (12a)-(12d). For these problems, $\psi$ is $r_{0}^{(k)}$ and $-r_{0}^{(k)}$ for the real pole, whereas for the complex-conjugate pole pairs, $\psi$ is selected to be $r_{\ell}^{(k)},-r_{\ell}^{(k)}, \theta_{\ell}^{(k)}$, and $-\theta_{\ell}^{(k)}$ for $\ell=1,2, \ldots, L_{0}^{(k)}+L_{1}^{(k)}$. 
In order to guarantee the stability of the resulting filters and to prevent the poles from changing their ordering, e.g., to inhibit the outermost complex-conjugate pole pair from becoming the second outermost complex-conjugate pole pair when minimizing its radius, the following additional constraints:

$$
-1 \leq r_{0}^{(1)} \leq r_{0}^{(2)} \leq \cdots \leq r_{0}^{(K)}<1
$$

and

$$
\begin{aligned}
0 & \leq r_{1}^{(1)} \leq r_{1}^{(2)} \leq \cdots \leq r_{1}^{(K)} \leq r_{L_{0}^{(1)}+1}^{(1)} \leq r_{L_{0}^{(2)}+1}^{(2)} \leq \cdots \leq r_{L_{0}^{(K)}+1}^{(K)} \\
& \leq r_{2}^{(1)} \leq r_{2}^{(2)} \leq \cdots \leq r_{2}^{(K)} \leq r_{L_{0}^{(1)}+2}^{(1)} \leq r_{L_{0}^{(2)}+2}^{(2)} \leq \cdots \leq r_{L_{0}^{(K)}+2}^{(K)} \leq \cdots \\
& \leq r_{L_{0}^{(1)}}^{(1)} \leq r_{L_{0}^{(2)}}^{(2)} \leq \cdots \leq r_{L_{0}^{(K)}}^{(K)} \leq r_{L_{0}^{(1)}+L_{1}^{(1)}}^{(1)} \leq r_{L_{0}^{(2)}+L_{1}^{(2)}}^{(2)} \leq \cdots \leq r_{L_{0}^{(K)}+L_{1}^{(K)}}^{(K)}<1
\end{aligned}
$$

are required. ${ }^{2}$

For later use, $\Phi_{1}^{(k)}$ and $\Phi_{2}^{(k)}$ denote the solutions with minimized $r_{0}^{(k)}$ and $-r_{0}^{(k)}$ (maximized $\left.r_{0}^{(k)}\right)$, whereas

$$
\Phi_{2+\ell^{\prime}}^{(k)} \quad \Phi_{2+\left(L_{0}^{(k)}+L_{1}^{(k)}\right)+\ell^{\prime}}^{(k)} \quad \Phi_{2+2\left(L_{0}^{(k)}+L_{1}^{(k)}\right)+\ell^{\prime}}^{(k)} \text { and } \quad \Phi_{2+3\left(L_{0}^{(k)}+L_{1}^{(k)}\right)+\ell}^{(k)}
$$

for $\ell=1,2, \ldots, L_{0}^{(k)}+L_{1}^{(k)}$ denote the solutions with the minimized $r_{\ell}^{(k)}$, the minimized $-r_{\ell}^{(k)}$ (maximized $r_{\ell}^{(k)}$ ), the minimized $\Theta_{\ell}^{(k)}$, and the minimized $-\Theta_{\ell}^{(k)}$ (maximized $\Theta_{\ell}^{(k)}$ ), respectively.

To solve these problems, the passband and stopband regions in the magnitude criteria of (12a)-(12d) are discretized into the frequency points $\omega_{i} \in \Omega_{p}$ for $i=1,2, \ldots, \Xi_{p}$ and $\omega_{i} \in \Omega_{S}$ for $i=\Xi_{p}+1, \Xi_{p}+2, \ldots, \Xi_{p}+\Xi_{s}$, which gives rise to the following discretized criteria:

$$
\left|E\left(\Phi, \omega_{i}\right)\right|-1 \leq 0 \quad \text { for } i=1,2, \ldots, \Xi_{p}+\Xi_{S}
$$

and

$$
E\left(\Phi, \omega_{i}\right) \leq 0 \text { for } i=1,2, \ldots, \Xi_{p}
$$

The resulting discrete minimization problems are to find $\Phi$ to minimize $\psi$ subject to the constraints of (20a) and (20b) and the constraints of (21a) and (21b). Here, $\psi$ is one of the abovementioned $2+4\left(L_{0}^{(k)}+L_{1}^{(k)}\right)$ problems for each of the $K$ sub-stages, that is, the total number

\footnotetext{
${ }^{2}$ In these constraints, it is assumed that the following two facts are valid. First, the transfer function, as given by (1), (2a), (2b), (3a), and (3b), is either a low-pass or high-pass filter design. Second, the orders of $K$ subfilters, as given by $2\left(L_{0}^{(k)}+L_{1}^{(k)}\right)+1$ for $k=1,2, \ldots, K$ are the same, denoted by $2 \widetilde{L}+1$ so that each stage has $\widetilde{L}$ complex-conjugate pole-pairs. Under these assumptions, (20a) means that the radius of the real pole for the $(k+1)$ th stage is larger than that for the $k$ th stage for $k=1,2, \ldots, K-1$. According to (20b), the same is true when considering the radii of the innermost complex-conjugate pole pairs included in the $K$ sub-stages. Furthermore, this fact is valid up to the $\widetilde{L}$ th innermost pole pairs (that are simultaneously the outmost pole pairs) in these sub-stages. In addition, (20b) implies that the radius of the second innermost complex-conjugate pole pair in the first stage is larger than the radius of the innermost complex-conjugate pole pair in the last stage and the same constraint is true up to the $\widetilde{L}$ th innermost pole pairs.
} 
of problems is

$$
\sum_{k=1}^{K}\left[2+4\left(L_{0}^{(k)}+L_{1}^{(k)}\right)\right]
$$

The above-mentioned problems can be conveniently solved by using the second algorithm of Dutta and Vidyasagar (Dutta \& Vidyasagar, 1977) or the function fmincon from the optimization toolbox provided by MathWorks, Inc. (Coleman et al., 1999). For more detail, see (Saramäki \& Yli-Kaakinen, 2002; Yli-Kaakinen, 2002; Yli-Kaakinen \& Saramäki, 2007).

For transfer functions, as given by (1), (2a), (2b), (3a), and (3b), the key goal is to quantize the adaptor coefficients $\gamma_{\ell}^{(k)}$ for $\ell=0,1, \ldots, 2\left(L_{0}^{(k)}+L_{1}^{(k)}\right)$ and for $k=1,2, \ldots, K$ to achieve the optimization target stated in Section 4 . It can be shown that the larger region including the feasible region, where LWD filter meets the given criteria, can be determined, by means of the above solutions $\Phi_{p}^{(k)}$ for $p=1,2, \ldots, 2+4\left(L_{0}^{(k)}+L_{1}^{(k)}\right)$ and for $k=1,2, \ldots, K$, by specifying the minimum and maximum values of $\gamma_{\ell}^{(k)}$ for $\ell=0,1, \ldots, 2\left(L_{0}^{(k)}+L_{1}^{(k)}\right)$ and for $k=1,2, \ldots, K$ as follows:

$$
\gamma_{\ell}^{(k)(\min )}=\min _{p=1,2, \ldots, 2+4\left(L_{0}^{(k)}+L_{1}^{(k)}\right)}\left\{\gamma_{\ell, p}^{(k)}\right\} \text { and } \gamma_{\ell}^{(k)(\max )}=\max _{p=1,2, \ldots, 2+4\left(L_{0}^{(k)}+L_{1}^{(k)}\right)}\left\{\gamma_{\ell, p}^{(k)}\right\} \text {, }
$$

where $\gamma_{\ell, p}^{(k)}$ denotes the value of $\gamma_{\ell}^{(k)}$ determined according to the $p$ th solution, $\Phi_{p}^{(k)}$, of the above-mentioned optimization problems.

As shown in Fig. 8(a), the search space determined in the above manner by the adaptor coefficient values for the complex-conjugate pole pairs is significantly larger than the corresponding original space found in terms of the radius and the angle for the pole pair under consideration. When concentrating in the sequel on determining desired finite-precision values for the adaptor coefficients, the use of the smaller search space will be utilized in a manner to be described later on in Subsection 5.3.4.

\subsubsection{Approximately linear-phase LWD Filters}

When determining the smallest and largest radius of the real pole and the smallest and largest values of the radius and the angle for each of the complex-conjugate pole pairs for the approximately linear-phase LWD filters, there are two main differences compared to the cascaded LWD filters. First, the overall filter is constructed as a single stage, that is, $K=1$. Therefore, the constraints of (20a) and (20b) reduce, in the low-pass case, to the constraints that all the radii are less than unity and the complex-conjugate pole pairs are ordered in terms of their radii such that their ordering remains intact. Second, in addition to the above-mentioned constraints on the radii of the poles and the magnitude-response constraints of (21a) and (21b), the following phase-response constraints:

$$
\left|\arg H\left(\Phi, \mathrm{e}^{\mathrm{j} \omega_{i}}\right)-\tau \omega_{i}\right|-\Delta \leq 0 \text { for } i=1,2, \ldots, \Xi_{p}
$$

should be included. These constraints are obtained from the original phase response constraint, as given by (15) in Subsection 4.2, by dicretizing the passband region into the frequency points $\omega_{i} \in \Omega_{p}$ for $i=1,2, \ldots, \Xi_{p}$ in a manner similar to that performed earlier for the magnitude criteria. 


\subsubsection{Recursive $N$ th-band decimators and interpolators}

For recursive $N$ th-band decimators and interpolators, there are also two differences compared to the cascaded LWD filters when determining the parameter space of the infinite-precision coefficients. First, the transfer functions, as given by (8a), (8b), and (8c), have only real poles and, therefore, the number of problems reduces to $2 \sum_{n=0}^{N_{k}-1} L_{n}^{(k)}$ for each of the $K$ sub-stages. For these problems, $\psi$ is $r_{\ell}^{(k)}$ and $-r_{\ell}^{(k)}$ for $\ell=1,2, \ldots, L_{0}^{(k)}+L_{1}^{(k)}+\cdots+L_{N_{k}-1}^{(k)}$ and for $k=1,2, \ldots, K$. In this case,

$$
\Phi_{\ell}^{(k)} \quad \text { and } \quad \Phi_{L_{0}^{(k)}+L_{1}^{(k)}+\cdots+L_{N_{k}-1}^{(k)}+\ell}^{(k)}
$$

for $\ell=1,2, \ldots, L_{0}^{(k)}+L_{1}^{(k)}+\cdots+L_{N_{k}-1}^{(k)}$ denote the solutions with minimized $r_{\ell}^{(k)}$ and $-r_{\ell}^{(k)}$ (maximized $r_{\ell}^{(k)}$ ), respectively. The above procedure gives for each real pole $r_{\ell}^{(k)}$ for $\ell=$ $1,2, \ldots, L_{0}^{(k)}+L_{1}^{(k)}+\cdots+L_{N_{K}-1}^{(k)}$ and for $k=1,2, \ldots, K$, the region $r_{\ell}^{(k)(\min )} \leq r_{\ell}^{(k)} \leq r_{\ell}^{(k)(\max )}$ that is directly the feasible region, where the pole can be located such that by relocating the remaining poles the given overall criteria are still met by using the infinite-precision arithmetic. Second, the constraints of (20a) and (20b) for the radii of the real poles and for the complexconjugate pole pairs are replaced by the following constraints for radii of the real poles:

$$
\begin{aligned}
-1 & \leq r_{1}^{(k)} \leq r_{L_{0}^{(k)}+1}^{(k)} \leq \cdots \leq r_{L_{0}^{(k)}+L_{1}^{(k)}+\cdots+L_{N_{1}-2}^{(k)}+1}^{(k)} \\
& \leq r_{2}^{(k)} \leq r_{L_{0}^{(k)}+2}^{(k)} \leq \cdots \leq r_{L_{0}^{(k)}+L_{1}^{(k)}+\cdots+L_{N_{1}-2}^{(k)}+2}^{(k)} \leq \cdots \leq \\
& \leq r_{L_{0}^{(k)}}^{(k)} \leq r_{L_{0}^{(k)}+L_{1}^{(k)}}^{(k)} \leq \cdots \leq r_{L_{0}^{(k)}+L_{1}^{(k)}+\cdots+L_{N_{1}-1}^{(k)}}^{(k)} \leq 0,
\end{aligned}
$$

for $k=1,2 \ldots, K^{3}$

\footnotetext{
${ }^{3}$ In this constraint, each of the $K$ sub-stages is considered independently of each other due to their own predetermined frequency-response shaping responsibilities in providing the desired overall magnitude response (Renfors \& Saramäki, 1987) in contrast to the cascaded LWD filters, where all the filter stages generate as joint effort the overall response in the same passband and stopband regions. For the $k$ th stage for $k=1,2, \ldots, K$, the above constraint simply means the following four experimentally observed facts. First, all the poles are located on the negative real axis. Second, if the overall number of adjustable poles in the $k$ th stage is $T_{1} N_{k}+T_{2}$, where $N_{k}$ is the decimation factor after this stage and $T_{1}$ and $T_{2}$ are integers, then the $n$th all-pass filter transfer function $A_{n}^{(k)}(z)$, which is involved in generating the $k$ th stage in the single-stage equivalent in Section 2.3 according to (8a), (8b), and (8c), contains $T_{1}+1$ and $T_{1}$ adjustable real pole locations for $n=0,1, \ldots, T_{2}-1$ and for $n=T_{2}, T_{2}+1, \ldots, N_{k}-1$, respectively. Third, when considering the radii of the outermost poles in the above-mentioned all-pass filter transfer functions for $n=0,1, \ldots, T_{2}-1$, the radius of the $n$th transfer function is less than that of $(n+1)$ th transfer function. Fourth, if $T_{1}>1$ and it is assumed that the outermost real pole is absent for $n=$ $T_{2}, T_{2}+1, \ldots, N_{k}-1$, then the following two additional facts are true. First, the above-mentioned third fact is true starting from the second outermost real poles up to the innermost real pole for $n=$ $0,1, \ldots, N_{k}-1$. Second, if the location of the pole of the last transfer function is more innermost than that of first transfer function, then its radius is smaller.
} 


\subsection{Optimization of Finite-Precision Filters}

It has been experimentally proved that the above-defined parameter space for each of three filter types under consideration forms a space including the feasible space where the filter specifications are satisfied. After finding this larger space, all that is needed is to check whether in this space there exist combinations of the discrete pole positions with which the given overall criteria are met.

\subsubsection{Cascade connection of LWD filters}

For cascade connections of low-order LWD filters, this search can be conveniently accomplished by first finding the sets of powers-of-two numbers $\Gamma_{\ell}^{(k)}$ for $\ell=0,1, \ldots, 2\left(L_{0}^{(k)}+L_{1}^{(k)}\right)$ and for $k=1,2, \ldots, K$ between the smallest and largest values of each adaptor coefficient, that is, by determining

$$
\left\{\Gamma_{\ell}^{(k)} \in \mathbb{P O T}_{\left(R, P_{R}\right)} \mid \gamma_{\ell}^{(k)(\min )} \leq \Gamma_{\ell} \leq \gamma_{\ell}^{(k)(\max )}\right\}
$$

for $\ell=0,1, \ldots, 2\left(L_{0}^{(k)}+L_{1}^{(k)}\right)$ and for $k=1,2, \ldots, K$. Here, $\mathbb{P O T}_{\left(R, P_{R}\right)}$ denotes the space of the powers-of-two numbers for $R$, the given maximum number of power-of-two terms, and $P_{R}$, the maximum number of fractional bits [cf. (9)]. Denote by $S_{\ell}^{(k)}$ the number of powers-of-two values between $\gamma_{\ell}^{(k)(\min )}$ and $\gamma_{\ell}^{(k)(\max )}$. Furthermore, denote by $\Gamma_{\ell}^{(k)(s)}$ for $s=1,2, \ldots, S_{\ell}^{(k)}$ the sth existing discrete value between these smallest and largest values.

The magnitude response is then evaluated for each combination of the $\Gamma_{\ell}^{(k)(s)}$ for $\ell=$ $0,1, \ldots, 2\left(L_{0}^{(k)}+L_{1}^{(k)}\right)$ and $s=1,2, \ldots, S_{\ell}^{(k)}$ to check whether the filter meets the given specifications. Hence, the number of discrete coefficient value combinations to be considered is

$$
\prod_{k=1}^{K} \prod_{\ell=0}^{2\left(L_{0}^{(k)}+L_{1}^{(k)}\right)} S_{\ell}^{(k)} .
$$

\subsubsection{Approximately linear-phase LWD Filters}

For approximately linear-phase LWD filters, the phase response is evaluated for all the solutions satisfying the magnitude specifications to make sure that the finite-wordlength filter meets the given overall criteria, that is, also the phase criteria of (23).

\subsubsection{Recursive $N$ th-band decimators and interpolators}

For multistage decimators and interpolators, this finite-precision search can be performed independently for each filter stage as in the single-stage equivalent described in Subsection 2.3, all the filter stages have, according to the discussion in (Renfors \& Saramäki, 1987), their own roles in providing the given attenuation in the predetermined stopband regions. This considerably reduces the overall optimization time. Furthermore, having only real poles in the overall implementation significantly reduces the overall finite-precision optimization time.

\subsubsection{Finite wordlength considerations}

The proper values for $R$ and $P_{R}$ are selected to be the smallest values for which there exist the discrete coefficient values between the smallest and largest values for the adaptor coefficients. If no solution satisfying the prescribed criteria are found for the predetermined discrete coefficient representation form, then another less stringent coefficient representation has to be 
tried, that is, the wordlength or the maximum number of power-of-two terms is gradually increased and the search is restarted until one or more desired finite-precision filters meeting the given specifications are found.

It should be pointed out that for certain given wordlengths, there are typically several solutions meeting the magnitude specifications. Therefore, it is advisable to find first all the solutions satisfying the given criteria and then to choose among which the one with the best attenuation characteristics or the minimum number of adders and/or subtracters required to implement all the multipliers for the given wordlength.

In Fig. 8, the dots indicate the allowable locations for both the upper-half-plane complexconjugate pole and a real pole when three power-of-two terms with seven fractional bits are used for the adaptor coefficient representations $\left(R=3\right.$ and $\left.P_{R}=7\right)$. Note that these distributions are highly irregular for a few power-of-two terms due to the desired coefficient representation form. However, as can be seen from this figure, there are, particularly for the innermost complex-conjugate pole, regions where the angle of the pole corresponding to finite-precision values of $\gamma_{2 l-1}$ and $\gamma_{2 l}$ is smaller than $\Theta^{(\min )}$ or larger than $\Theta^{(\max )}$. For this reason, it is advisable to check whether the angle of the discrete pole is in the prescribed region in order to avoid the vain evaluation of the corresponding magnitude response. In addition, it is beneficial, in order to speed up the search, to check whether the filter meets the given magnitude specifications in two steps. First, the magnitude response is evaluated at band edges, that is, in the low-pass case at $\omega=\omega_{p}$ and at $\omega=\omega_{s}$. Second, only if the magnitude response at these points stays within the given specifications, the remaining frequency points are evaluated. This is because the worst-case deviations in both the passband(s) and stopband(s) of the resulting finite-precision filter occur most likely at the band edges.

\section{Numerical Examples}

This section shows, by means of examples, the applicability of the overall synthesis scheme described in the previous section for solving three optimization problems stated in Section 4. More examples can be found in (Yli-Kaakinen, 1998; 2002; Yli-Kaakinen \& Saramäki, 1999a;b; 2000; 2005; 2007).

\subsection{Example 1}

This example is included to illustrate the performance of the proposed overall synthesis scheme for designing cascade connections of low-order LWD filters as well as to show the superiority of these cascaded filters over direct LWD filters in finite wordlength implementations.

It is desired to design a low-pass filter with the passband and stopband edges at $\omega_{p}=0.1 \pi$ and at $\omega_{s}=0.2 \pi$, respectively. The maximum allowable passband ripple is $A_{p}=0.5 \mathrm{~dB}$ $\left(\delta_{p}=0.0559\right)$ and the minimum stopband attenuation is at least $A_{s}=100 \mathrm{~dB}\left(\delta_{s}=10^{-5}\right)$, respectively.

When the three-stage quantization scheme described in Section 5 is applied to $K=4$, that is, the overall transfer function is a cascade of four LWD filters of the same order, the initial infinite-precision start-up solution for further optimization described in Subsection 5.1.1 (the first main step of Section 5) can be determined by using four identical copies of a third-order elliptic filter with the passband ripple of $\delta_{p} / 4=0.0143$ and the stopband ripple of $\sqrt[4]{\delta_{s}}=$ 0.0562. The minimum odd order of an elliptic filter to meet the given magnitude criteria is three. For this third-order initial elliptic subfilter just meeting the given passband criteria, the minimum stopband attenuation is $25.75 \mathrm{~dB}\left(\delta_{s}=0.05158\right)$. The radius of the real pole as well 


\begin{tabular}{|c|c|}
\hline$A_{0}^{(1,2,3,4)}(z)$ & $A_{1}^{(1,2,3,4)}(z)$ \\
\hline$r_{0}^{(1,2,3,4)}=0.714855$ & $r_{1}^{(1,2,3,4)}=0.893594 \quad \theta_{1}^{(1,2,3,4)}=0.118835 \pi$ \\
\hline
\end{tabular}

Table 1. Initial pole locations for the cascade of four LWD filters in Example 1.

as the radius and positive angle of the complex-conjugate pole pair for these initial subfilters are given in Table 1. This initial filter already meets the given magnitude specifications and can, therefore, be used itself without further optimization for accomplishing the second main step of Section 5 that is described for these cascaded LWD filters in Subsection 5.2.1.

The smallest and largest values of the adaptor coefficients after the infinite-precision optimization of this subsection are included in Table 2. In addition, this table gives the smallest and largest values of the adaptor coefficients quantized at the third main step of Section 5 that is described for these filters in Section 5.3.1 to the three power-of-two terms and five fractional bits $\left(R=3\right.$ and $\left.P_{R}=5\right){ }^{4}$ The number of admissible discrete values $S_{\ell}^{(k)}$ between $\gamma_{\ell}^{(k)(\min )}$ and $\gamma_{\ell}^{(k)(\min )}$ for $\ell=0,1,2$ and for $k=1,2,3,4$ are also summarized in this table. In this case, the overall number of combinations to be evaluated is approximately $134 \cdot 10^{6}$ [cf. (26)]. The CPU time required by a Fortran 95 program to evaluate all these finite-precision coefficient combinations on a 1.4-GHz Pentium-M with $\Xi_{p}=\Xi_{s}=30$ [cf. (21a) and (21b)] was approximately 400 seconds.

The search space after the infinite-precision optimization is depicted in Fig. 9. In this figure, the circles indicate the allowable locations for the poles inside the search space for the abovementioned adaptor coefficient representation form, whereas the largest, the second largest, the third largest, and the smallest search spaces correspond to the $k$ th sub-stage for $k=1$, $k=2, k=3$, and $k=4$, respectively.

The specifications are met by the adaptor coefficients given in Table 3. A total of only six adders and/or subtracters are required to implement all the adaptor coefficients when the adaptors shown in Fig. 6 are used. Note that two sub-stages are identical. For this coefficient representation form, there are 17 finite-precision solutions meeting the specifications among which the one with the minimum implementation cost is selected. In Figure 9, the crosses denote the pole locations of this optimal solution. Figure 10 shows for this design the magnitude responses of the four sub-stages as well as that of the overall filter. In addition, the passband details of the magnitude response for the overall filter is included in this figure. The pole-zero plot for the overall design is depicted in Fig. 11.

For $K=1$, in turn, that is, for the single-stage design, the given criteria are met by the ninthorder filter with adaptor coefficients given in Table 4 . In this case, four power-of-two terms with nine fractional bits $\left(R=4\right.$ and $\left.P_{R}=9\right)$ are required by the adaptor coefficients to still meet the magnitude criteria. The magnitude responses and the pole-zero plot for this direct LWD design are depicted in Figs. 12 and 13, respectively.

The above cascade of four low-order LWD filter sections is very attractive for VLSI implementations because the use of a costly multiplier element can be replaced by a harwired logic. If the adaptors of Fig. 6 are utilized, then this harwired logic requires at most two power-of-two

\footnotetext{
${ }^{4}$ In this case, three power-of-two terms and four fractional bits $\left(R=3\right.$ and $\left.P_{R}=4\right)$ is the shortest wordlength for which there exist at least one discrete value between the smallest and largest values of each adaptor coefficient. However, for this coefficient wordlength, there is no solution satisfying the given specifications.
} 


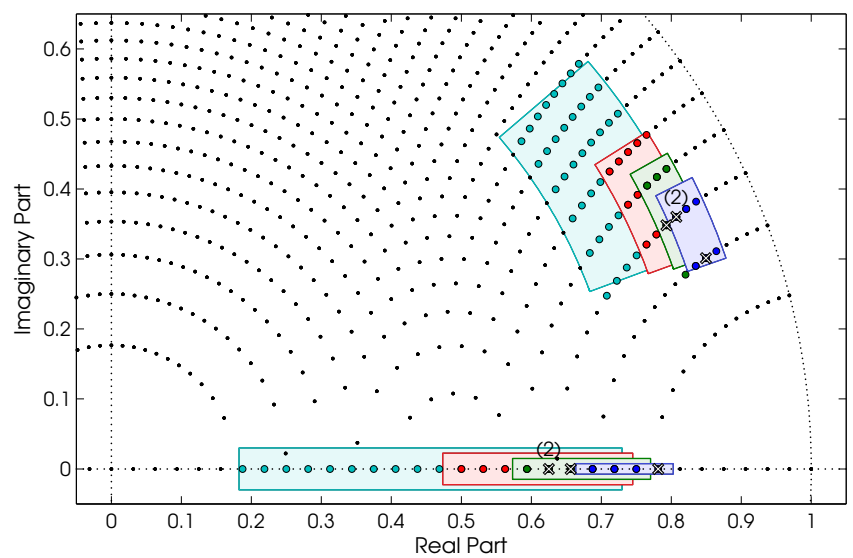

Fig. 9. Search spaces for the cascade of four LWD filters in Example 1 in the $R=3$ and $P_{R}=5$ case.

\begin{tabular}{rrrrccc}
\hline \multirow{2}{*}{$k$} & $\ell$ & $\gamma_{\ell}^{(k)(\min )}(z)$ & $\gamma_{\ell}^{(k)(\max )}(z)$ & $\Gamma_{\ell}^{(k)(1)}(z)$ & $\Gamma_{\ell}^{(k)\left(S_{\ell}^{(k)}\right)}(z)$ & $S_{\ell}^{(k)}$ \\
\hline \multirow{4}{*}{1} & 0 & 0.182392 & 0.729620 & $2^{-2}-2^{-4}$ & $1-2^{-2}-2^{-5}$ & 18 \\
& 1 & -0.802832 & -0.531560 & $-1+2^{-2}-2^{-5}$ & $-2^{-1}-2^{-4}$ & 8 \\
& 2 & 0.739326 & 0.931286 & $1-2^{-2}$ & $1-2^{-3}+2^{-5}$ & 6 \\
\hline \multirow{4}{*}{2} & 0 & 0.473568 & 0.745019 & $2^{-1}$ & $1-2^{-2}-2^{-5}$ & 8 \\
& 1 & -0.817631 & -0.666228 & $-1+2^{-2}-2^{-4}$ & $-1+2^{-2}+2^{-4}$ & 5 \\
& 2 & 0.835625 & 0.934313 & $1-2^{-3}-2^{-5}$ & $1-2^{-3}+2^{-5}$ & 3 \\
\hline & 0 & 0.573298 & 0.770266 & $2^{-1}+2^{-3}-2^{-5}$ & $1-2^{-2}$ & 6 \\
3 & 1 & -0.834543 & -0.726433 & $-1+2^{-2}-2^{-4}$ & $-1+2^{-2}$ & 3 \\
& 2 & 0.863579 & 0.937735 & $1-2^{-3}$ & $1-2^{-4}$ & 3 \\
\hline & 0 & 0.663425 & 0.802724 & $1-2^{-2}-2^{-4}$ & $1-2^{-2}+2^{-5}$ & 4 \\
4 & 1 & -0.861770 & -0.757413 & $-1+2^{-3}+2^{-5}$ & $-1+2^{-2}-2^{-5}$ & 3 \\
& 2 & 0.887134 & 0.942355 & $1-2^{-3}+2^{-5}$ & $1-2^{-4}$ & 2 \\
\hline
\end{tabular}

Table 2. The smallest and largest values for both the infinite-precision and finite-precision coefficients in Example 1.

terms, instead of $R=3$ terms, containing only $P_{R}=5$ fractional for implementing all the $\alpha$ values in these adaptors.

In comparison, the direct LWD design requires for some coefficient values $R=4$ power-oftwo terms and $P_{R}=9$ fractional bits. The price paid for this significantly reduced complexity in implementing the adaptor coefficient values in the cascaded implementation is a slight increase (from nine to twelve) in the overall filter order compared to the direct LWD filter. Another remarkable advantage of the proposed cascaded filter in comparison with the direct LWD filter is that the radius of the outermost complex-conjugate pole pair is significantly 


\begin{tabular}{llll}
\hline \multicolumn{1}{c}{$A_{0}^{(k)}(z)$} & \multicolumn{2}{c}{$A_{1}^{(k)}(z)$} \\
\cline { 1 - 3 } \cline { 5 - 6 }$\gamma_{0}^{(1,2)}=2^{-1}+2^{-3}$ & & $\gamma_{1}^{(1,2)}=-1+2^{-2}-2^{-5}$ & $\gamma_{2}^{(1,2)}=1-2^{-3}+2^{-5}$ \\
$\gamma_{0}^{(3)}=2^{-1}+2^{-3}+2^{-5}$ & $\gamma_{1}^{(3)}=-1+2^{-2}$ & $\gamma_{2}^{(3)}=1-2^{-3}+2^{-5}$ \\
$\gamma_{0}^{(4)}=1-2^{-2}+2^{-5}$ & $\gamma_{1}^{(4)}=-1+2^{-2}-2^{-4}$ & $\gamma_{2}^{(4)}=1-2^{-4}$ \\
\hline
\end{tabular}

Table 3. Optimized finite-precision adaptor coefficients for the cascade of four LWD filters in Example 1.

\begin{tabular}{cll}
\hline \multicolumn{1}{c}{$A_{0}^{(0)}(z)$} & & $A_{1}^{(1)}(z)$ \\
\cline { 1 - 1 }$\gamma_{0}^{(1)}=1-2^{-3}+2^{-6}$ & & \\
$\gamma_{1}^{(1)}=-1+2^{-3}+2^{-6}+2^{-9}$ & $\gamma_{5}^{(1)}=-1+2^{-2}-2^{-4}+2^{-9}$ \\
$\gamma_{2}^{(1)}=1-2^{-5}$ & $\gamma_{6}^{(1)}=1-2^{-6}+2^{-9}$ \\
$\gamma_{3}^{(1)}=-1+2^{-5}-2^{-7}-2^{-9}$ & $\gamma_{7}^{(1)}=-1+2^{-4}+2^{-6}$ \\
$\gamma_{4}^{(1)}=1-2^{-4}-2^{-8}$ & $\gamma_{8}^{(1)}=1-2^{-4}+2^{-6}-2^{-8}$ \\
\hline
\end{tabular}

Table 4. Optimized finite-precision adaptor coefficients for the direct LWD filter in Example 1.
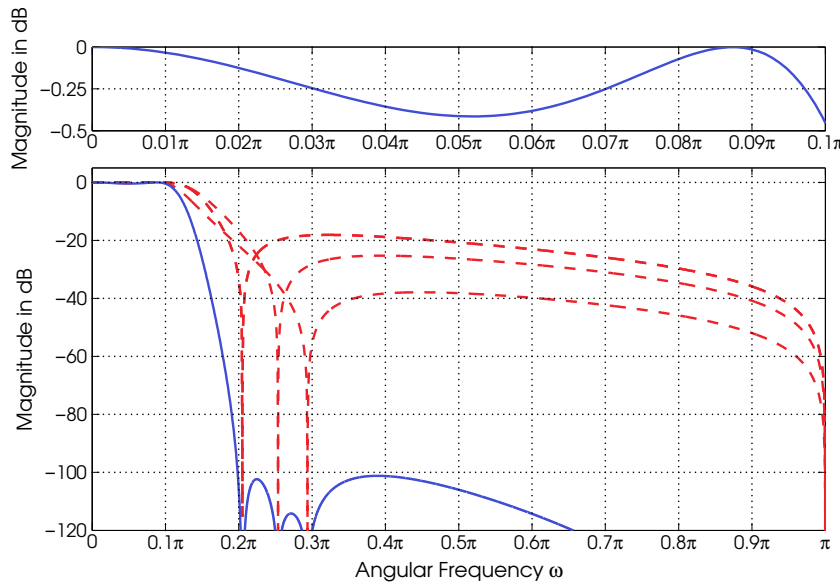

Fig. 10. Some magnitude responses for the cascade of four optimized finite-precision LWD filters in Example 1. The solid and dashed lines show the responses for the overall filter and the subfilters, respectively. Two subfilters are identical (the dashed line with the lowest attenuation).

smaller. For $K=1$ and $K=4$, these values are 0.98920 and 0.90138 , respectively. When using the adaptors shown in Fig. 6, the output noise gains are $31.9 \mathrm{~dB}$ and $21.8 \mathrm{~dB}$ for $K=1$ and $K=4$, respectively. This means that for $K=4$ roughly two fewer bits are required for the data representation to arrive at approximately the same output noise level as with the corresponding direct LWD filter. 


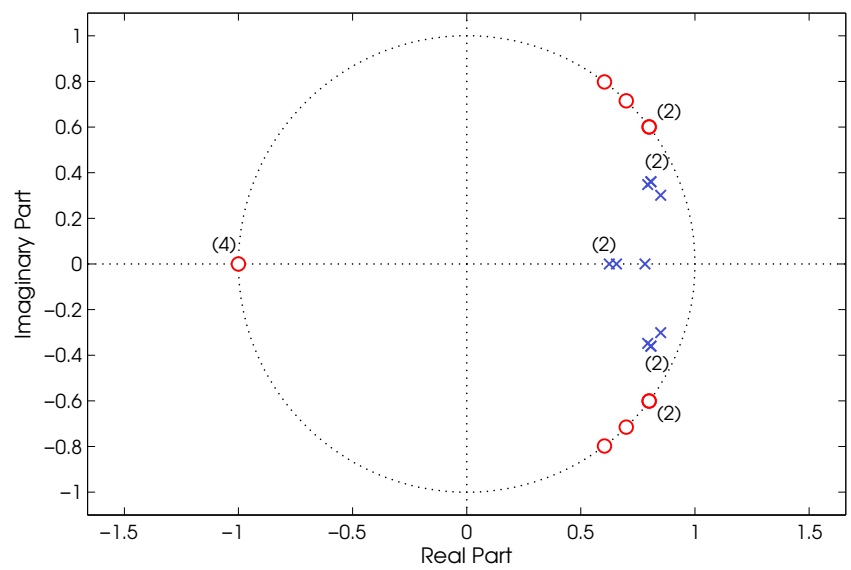

Fig. 11. Pole-zero plot for the cascade of four optimized finite-precision LWD filters in Example 1.

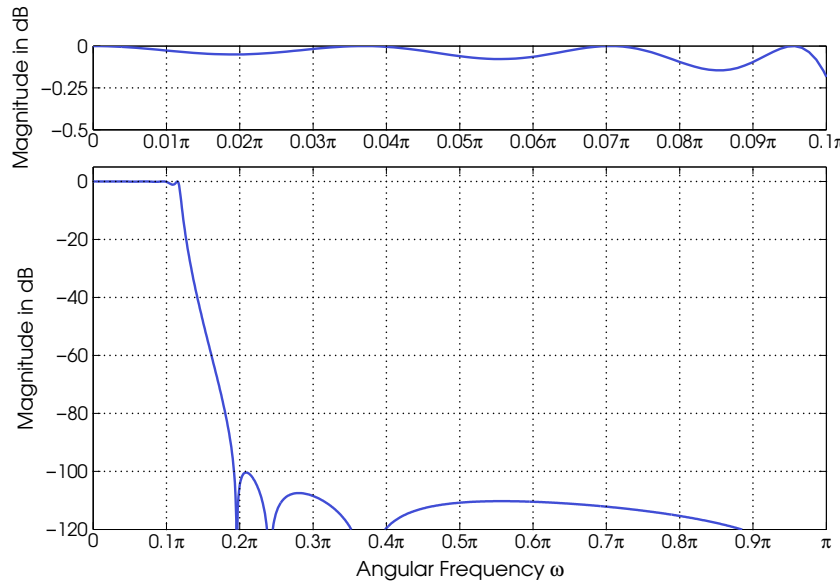

Fig. 12. Some magnitude responses for the optimized finite-precision direct LWD filter in Example 1.

\subsection{Example 2}

This example is included to illustrate the performance of the proposed overall synthesis scheme for designing approximately linear-phase finite-precision LWD filters as well as to compare these filters with their linear-phase FIR filter equivalents.

It is desired to design a low-pass filter with passband and stopband edges at $\omega_{p}=0.05 \pi$ and at $\omega_{s}=0.1 \pi$, respectively. The maximum allowable passband ripple is $A_{p}=0.2 \mathrm{~dB}\left(\delta_{p}=\right.$ $0.0228)$ and the stopband attenuation is $A_{s}=60 \mathrm{~dB}\left(\delta_{s}=10^{-3}\right)$. The maximum allowable phase deviation in the passband from the average slope, in turn, is $\Delta=0.5$ degrees. In this case, an excellent phase performance is obtained by using a ninth-order LWD filter. 


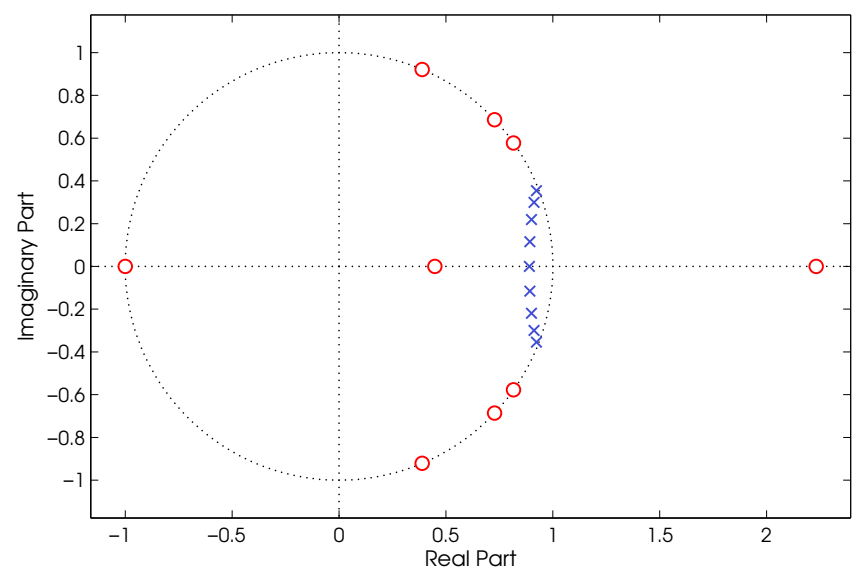

Fig. 13. Pole-zero plot for the optimized finite-precision direct LWD filter in Example 1.

\begin{tabular}{cll}
\hline \multicolumn{1}{c}{$A_{0}^{(1)}(z)$} & & $A_{1}^{(1)}(z)$ \\
\cline { 1 - 2 }$\gamma_{0}^{(1)}=1-2^{-4}$ & & \\
$\gamma_{1}^{(1)}=-1+2^{-5}-2^{-7}$ & & $\gamma_{5}^{(1)}=-1+2^{-4}+2^{-7}+2^{-9}$ \\
$\gamma_{2}^{(1)}=1-2^{-5}+2^{-7}$ & & $\gamma_{6}^{(1)}=1-2^{-6}-2^{-9}+2^{-11}$ \\
$\gamma_{3}^{(1)}=-1+2^{-3}-2^{-6}+2^{-10}$ & $\gamma_{7}^{(1)}=-1+2^{-3}-2^{-8}$ \\
$\gamma_{4}^{(1)}=1-2^{-7}-2^{-10}$ & $\gamma_{8}^{(1)}=1-2^{-8}$ \\
\hline
\end{tabular}

Table 5. Optimized finite-precision adaptor coefficients for the approximately linear-phase LWD filter in Example 2.

The filter specifications are met if the adaptor coefficient are represented using four powerof-two terms with eleven fractional bits $\left(R=4\right.$ and $\left.P_{R}=11\right)$ as given in Table 5. A total of ten adders and/or subtracters are required to implement all the adaptor coefficients when the adaptors shown in Fig. 6 are utilized. The magnitude and phase characteristics of the resulting filter are depicted in Fig. 14, whereas Fig. 15 gives the pole-zero plot.

The minimum order of a linear-phase FIR filter to meet the same magnitude specifications is 107, requiring 107 delay elements and 54 multipliers when exploiting coefficient symmetry. The delay of the linear-phase FIR equivalent is 53.5 samples, whereas for the proposed recursive filter the delay is only 40.9 samples.

\subsection{Example 3}

This example is included to illustrate the performance of the proposed overall design algorithm for synthesizing recursive Nth-band decimators. It is desired to design an eighth-band $(N=8)$ filter with the passband edge at $\omega_{p}=0.0785 \pi=0.628 \pi / 8$. The minimum stopband attenuation is at least $A_{S}=60 \mathrm{~dB}\left(\delta_{S}=10^{-3}\right)$. In this case, the stopband region, as given by $(17)$, is $\Omega_{S}=[0.1715 \pi, 0.3285 \pi] \cup[0.4215 \pi, 0.5785 \pi] \cup[0.6715 \pi, 0.8285 \pi] \cup[0.9215 \pi, \pi]$, that is, the aliasing into to the transition band $[0.0785 \pi, 0.125 \pi]$ is allowed from the bands $[0.3285 \pi, 0.4215 \pi],[0.5785 \pi, 0.6715 \pi]$, and $[0.8285 \pi, 0.9215 \pi]$. 

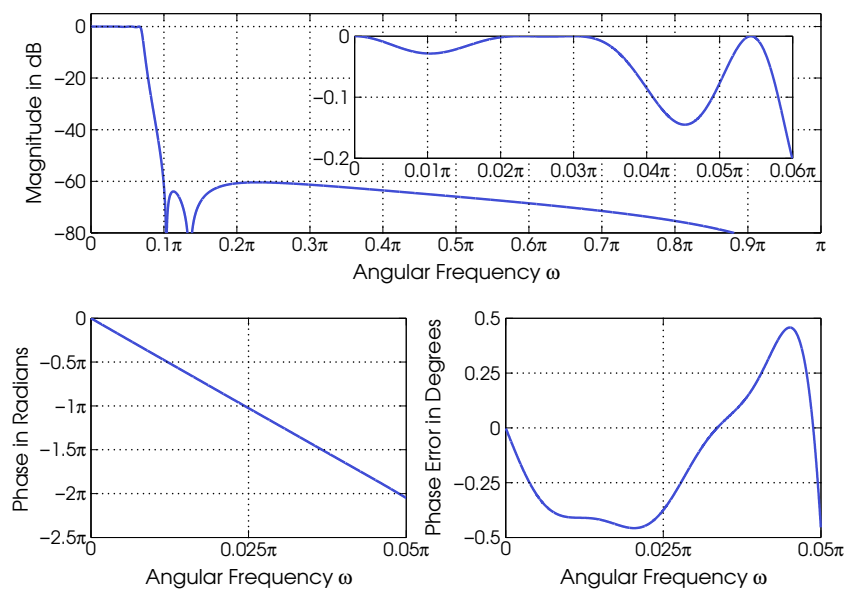

Fig. 14. Magnitude and phase responses for the optimized finite-precision approximately linear-phase LWD filter in Example 2.

For the three-stage design, the only option to factor the sampling rate conversion ratio is $N_{1}=N_{2}=N_{3}=2$. This factorization gives, according to the discussion of Subsection 2.3, rise to a single-stage equivalent with the transfer function of the form $H(z)=H_{1}(z) H_{2}\left(z^{2}\right) H_{3}\left(z^{4}\right)$ where $H_{1}(z), H_{2}(z)$, and $H_{3}(z)$ are half-band LWD filters. According to the design scheme described in (Renfors \& Saramäki, 1987), the desired 60-dB stopband attenuation is achieved by simultaneously determining these three subfilters such that $H_{3}\left(z^{4}\right), H_{2}\left(z^{2}\right)$, and $H_{1}(z)$ primarily take care of providing this attenuation on $[0.1715 \pi, 0.3285 \pi] \cup[0.6715 \pi, 0.8285 \pi]$, $[0.4215 \pi, 0.5785 \pi]$, and $[0.9215 \pi, \pi]$, respectively. The resulting minimum orders of $H_{1}(z)$, $H_{2}(z)$, and $H_{3}(z)$ to simultaneously meet the given specifications become 3,5 , and 7 , respectively. When following the notations of Subsection 2.3, the orders $L_{0}^{(k)}$ and $L_{1}^{(k)}$ of the branch transfer functions $A_{0}^{(k)}(z)$ and $A_{1}^{(k)}(z)$ of $H_{k}(z)$ for $k=1,2,3$ become $L_{0}^{(1)}=1$ and $L_{1}^{(1)}=0$; $L_{0}^{(2)}=L_{0}^{(2)}=1$; and $L_{0}^{(3)}=2$ and $L_{1}^{(3)}=1$; respectively.

The initial adaptor coefficient values for $H_{3}\left(z^{4}\right)$ are $\gamma_{1}^{(3)}=-0.085523, \gamma_{2}^{(3)}=-0.718273$, and $\gamma_{3}^{(3)}=-0.326452$, for $H_{2}\left(z^{2}\right), \gamma_{1}^{(2)}=-0.116797$ and $\gamma_{2}^{(2)}=-0.548630$, and for $H_{1}(z)$, $\gamma_{1}^{(1)}=-0.338473$. The stopband attenuations provided by these initial sub-stages $H_{3}\left(z^{4}\right)$, $H_{2}\left(z^{2}\right)$, and $H_{1}(z)$ in the stopband regions they primarily concentrate on are $73.21 \mathrm{~dB}, 83.97$ $\mathrm{dB}$, and $66.45 \mathrm{~dB}$, respectively. The smallest and largest values for the adaptor coefficients of the sub-stages $H_{3}\left(z^{4}\right), H_{2}\left(z^{2}\right)$, and $H_{1}(z)$ after applying the infinite-precision optimization of Subsection 5.2 are given in Table 6.

For this overall filter, the maximum number of power-of-two terms required to implement all the adaptor coefficients is four $(R=4)$, whereas eight fractional bits $\left(P_{R}=8\right)$ are required to meet the magnitude specifications. For this coefficient representation form, the number of discrete coefficient values between the smallest and largest values for the coefficients of $H_{3}\left(z^{3}\right)$ is 14,21 , and 33 , that is, the number of coefficient combinations for the last stage is $14 \cdot 21 \cdot 33=9702$. The number of discrete coefficient values between the smallest and largest values for the coefficients of $H_{2}\left(z^{2}\right)$ are 19 and 33, that is, the number of coefficient 


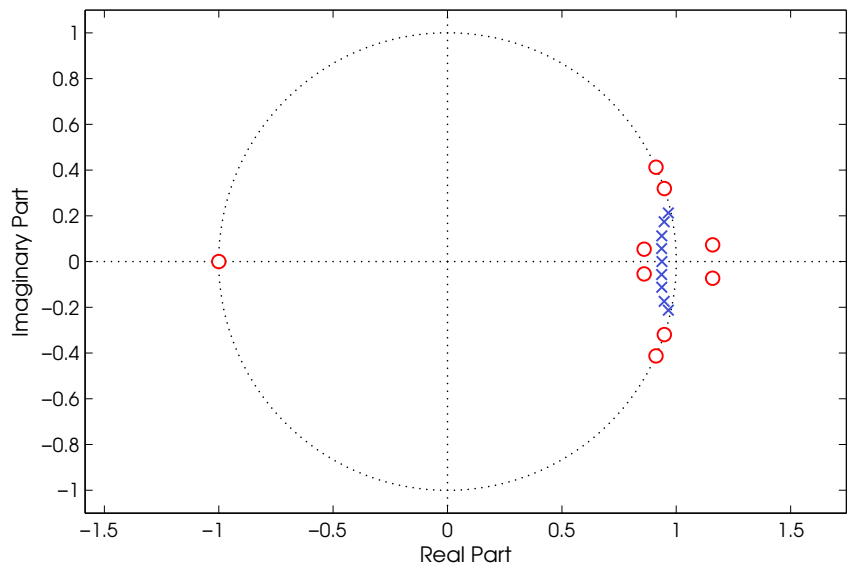

Fig. 15. Pole-zero plot for the optimized finite-precision approximately linear- phase LWD filter in Example 2.

\begin{tabular}{|c|c|c|c|c|c|}
\hline \multirow[t]{2}{*}{$H_{3}\left(z^{4}\right)$} & $A_{0}^{(3)}\left(z^{4}\right)$ & $\begin{array}{l}\gamma_{1}^{(3)(\min )} \\
\gamma_{2}^{(3)(\min )}\end{array}$ & $\begin{array}{l}=-0.111647 \\
=-0.771093\end{array}$ & $\begin{array}{l}\gamma_{1}^{(3)(\max )} \\
\gamma_{2}^{(3)(\max )}\end{array}$ & $\begin{array}{l}=-0.057811 \\
=-0.681117\end{array}$ \\
\hline & $A_{1}^{(3)}\left(z^{4}\right)$ & $\gamma_{3}^{(3)(\min )}$ & $=-0.395188$ & $\gamma_{3}^{(3)(\max )}$ & $=-0.268425$ \\
\hline \multirow{2}{*}{$\mathrm{H}_{2}\left(z^{2}\right)$} & $A_{0}^{(2)}\left(z^{2}\right)$ & $\gamma_{1}^{(2)(\min )}$ & $=-0.156770$ & $\gamma_{1}^{(2)(\max )}$ & $=-0.082365$ \\
\hline & $A_{1}^{(2)}\left(z^{2}\right)$ & $\gamma_{2}^{(2)(\min )}$ & $=-0.618978$ & $\gamma_{2}^{(2)(\max )}$ & $=-0.489915$ \\
\hline$H_{1}(z)$ & $A_{0}^{(1)}(z)$ & $\gamma_{1}^{(1)(\min )}$ & $=-0.341785$ & $\gamma_{1}^{(1)(\max )}$ & $=-0.336582$ \\
\hline
\end{tabular}

Table 6. The smallest and largest infinite-precision coefficient values for the subfilters $H_{3}\left(z^{4}\right)$, $H_{2}\left(z^{2}\right)$, and $H_{1}(z)$ in Example 3.

\begin{tabular}{lll}
\hline \multirow{3}{*}{$H_{3}\left(z^{4}\right)$} & $A_{0}^{(3)}\left(z^{4}\right)$ & $\begin{array}{l}\gamma_{1}^{(3)}=-0.07812500=-2^{-4}-2^{-6} \\
\gamma_{2}^{(3)}=-0.71093750=-1+2^{-2}+2^{-5}+2^{-7}\end{array}$ \\
\cline { 2 - 3 } & $A_{1}^{(3)}\left(z^{4}\right) \quad \gamma_{3}^{(3)}=-0.31250000=-2^{-2}-2^{-4}$ \\
\hline$H_{2}\left(z^{2}\right)$ & $A_{0}^{(2)}\left(z^{2}\right) \quad \gamma_{1}^{(2)}=-0.12500000=-2^{-3}$ \\
\cline { 2 - 3 } & $A_{1}^{(2)}\left(z^{2}\right) \quad \gamma_{2}^{(2)}=-0.56250000=-2^{-1}-2^{-4}$ \\
\hline$H_{1}(z)$ & $A_{0}^{(1)}(z)$ & $\gamma_{1}^{(1)}=-0.33984375=-2^{-1}+2^{-3}+2^{-5}+2^{-8}$ \\
\hline
\end{tabular}

Table 7. Optimized finite-precision coefficient values for the three-stage eighth-band filter in Example 3.

combinations for the second stage is 627 . For the first stage with transfer function $H_{1}(z)$, there exists only one discrete coefficient value between the smallest and largest values of the single coefficient. The CPU time required when using a Fortran 95 program on a $1.4 \mathrm{GHz}$ Pentium- 


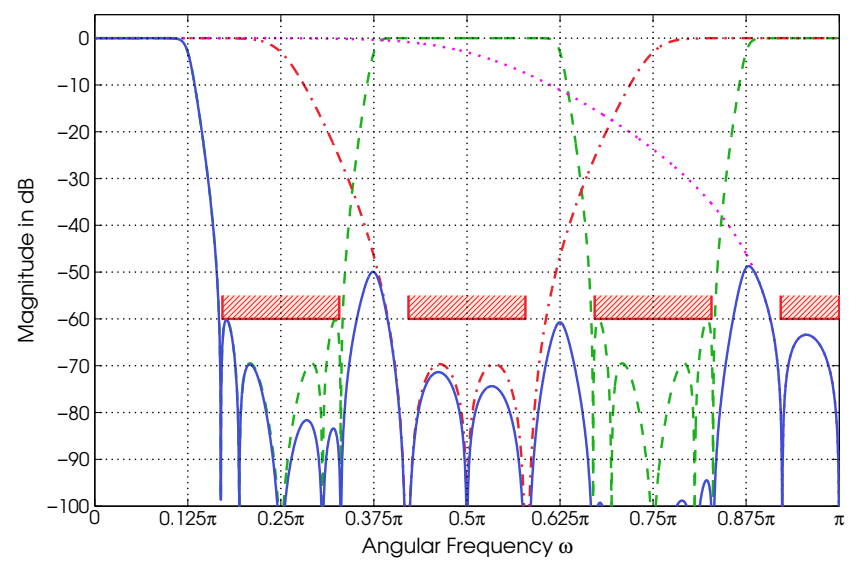

Fig. 16. Magnitude responses for the optimized finite-precision three-stage eighth-band decimator in Example 3. The solid line gives the magnitude response for the single-stage equivalent $H_{1}(z) H_{2}\left(z^{2}\right) H_{3}\left(z^{4}\right)$, whereas the dotted, dot-dashed, and dashed lines give the responses for $H_{1}(z), H_{2}\left(z^{2}\right)$, and $H_{3}\left(z^{4}\right)$, respectively.

\begin{tabular}{lll}
\hline$A_{0}^{(1)}(z)$ & $\gamma_{1}^{(1)}=-0.01953125=-2^{-6}-2^{-8}$ & $\gamma_{2}^{(1)}=-0.53125000=-2^{-1}-2^{-5}$ \\
$A_{1}^{(1)}(z)$ & $\gamma_{1}^{(1)}=-0.04687500=-2^{-4}+2^{-6}$ & $\gamma_{2}^{(1)}=-0.62500000=-2^{-1}-2^{-3}$ \\
$A_{2}^{(1)}(z)$ & $\gamma_{1}^{(1)}=-0.07812500=-2^{-4}-2^{-6}$ & $\gamma_{2}^{(1)}=-0.71875000=-1+2^{-2}+2^{-5}$ \\
$A_{3}^{(1)}(z)$ & $\gamma_{1}^{(1)}=-0.12109375=-2^{-3}+2^{-8}$ & $\gamma_{2}^{(1)}=-0.80859375=-1+2^{-2}-2^{-4}+2^{-8}$ \\
$A_{4}^{(1)}(z)$ & $\gamma_{1}^{(1)}=-0.17968750=-2^{-2}+2^{-4}+2^{-7}$ & $\gamma_{2}^{(1)}=-0.87890625=-1+2^{-3}-2^{-8}$ \\
$A_{5}^{(1)}(z)$ & $\gamma_{1}^{(1)}=-0.24218750=-2^{-2}+2^{-7}$ & $\gamma_{2}^{(1)}=-0.94921875=-1+2^{-4}-2^{-6}+2^{-8}$ \\
$A_{6}^{(1)}(z)$ & $\gamma_{1}^{(1)}=-0.32031250=-2^{-2}-2^{-4}-2^{-7}$ & \\
$A_{7}^{(1)}(z)$ & $\gamma_{1}^{(1)}=-0.43359375=-2^{-1}+2^{-4}+2^{-8}$ & \\
\hline
\end{tabular}

Table 8. Optimized finite-precision adaptor coefficients for the single-stage eighth-band decimator in Example 3.

$M$ to evaluate all these combinations with $\Xi_{s}=100$ stopband grid points was less than one second.

The number of adders and/or subtracters required to implement all the adaptor coefficients is seven when the adaptors shown in Fig. 6 are utilized. The optimized finite-precision coefficients values are given in Table 7 , whereas the magnitude responses for the sub-stages as well as for the single-stage equivalent are depicted in Fig. 16. The pole-zero plot for this equivalent is, in turn, shown in Fig. 17. The passband variation and the minimum stopband attenuation for the optimized finite-precision overall filter are $A_{p}=-4.278 \cdot 10^{-6} \mathrm{~dB}$ and $A_{s}=60.21 \mathrm{~dB}$, respectively. An efficient implementation of the optimized eight-band decimator is depicted in Fig. 18

For the single-stage design, that is, for a direct eighth-band filter, the minimum orders $L_{n}^{(1)}$ of the eight all-pass branch filters $A_{n}^{(1)}(z)$ for $n=0,1, \ldots, 7$ to meet the given specifications are $L_{n}^{(1)}=2$ for $n=0,1, \ldots, 5$ and $L_{6}^{(1)}=L_{7}^{(1)}=1$ so that the minimum number of multipli- 


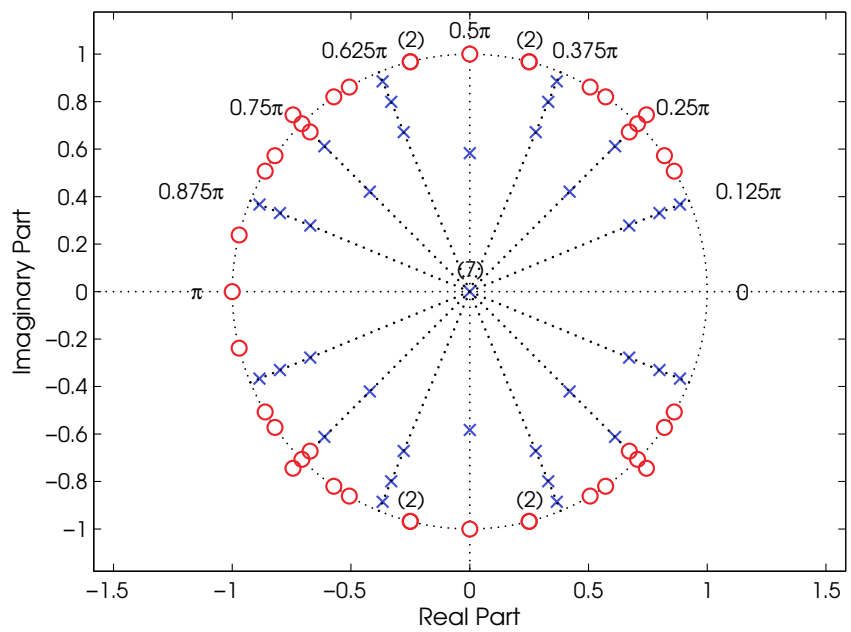

Fig. 17. Pole-zero plot for the optimized finite-precision three-stage eighth-band decimator in Example 3.

ers in the overall implementation is 14 . The stopband attenuation of this initial filter is 60.84 $\mathrm{dB}$. Again, the specifications are met by $R=4$ and $P_{R}=8$ even though the allowable margin for the coefficient quantization is only $0.84 \mathrm{~dB}$. The specifications are met by the adaptor coefficients given in Table 8. In this case, the number of adders and/or subtracters required to implement all the coefficients is 17 when the adaptors shown in Fig. 6 are utilized. The passband variation and the minimum stopband attenuation for this optimized finite-precision single-stage decimation filter are $A_{p}=1.584 \cdot 10^{-5} \mathrm{~dB}$ and $A_{s}=60.18 \mathrm{~dB}$, respectively. The magnitude response and the pole-zero plot for this decimation filter are depicted in Figs. 19 and 20 , respectively.

\section{Conclusions}

A systematic three-step algorithm has been developed for designing lattice wave digital (LWD) filters with short coefficient wordlength. The filter classes under consideration have been cascades of low-order LWD filters, approximately linear-phase LWD filters, and recursive Nth-band decimators and interpolators. The transfer functions, filter specifications, and optimization problems have been stated for each filter class under consideration. Then, the proposed three-step algorithm has been adapted for solving these optimization problems. The goal has been to find all the coefficient values such that the overall implementation does not require general multipliers. It has been shown that significant savings in the implementation cost are achieved by using the proposed technique. The efficiency and the robustness of the proposed algorithm has been demonstrated by means of several examples.

\section{References}

Ansari, R. \& Liu, B. (1983). Efficient sampling rate alteration using recursive IIR digital filters, IEEE Trans. Acoust., Speech, Signal Processing ASSP-31: 1366-1373. 


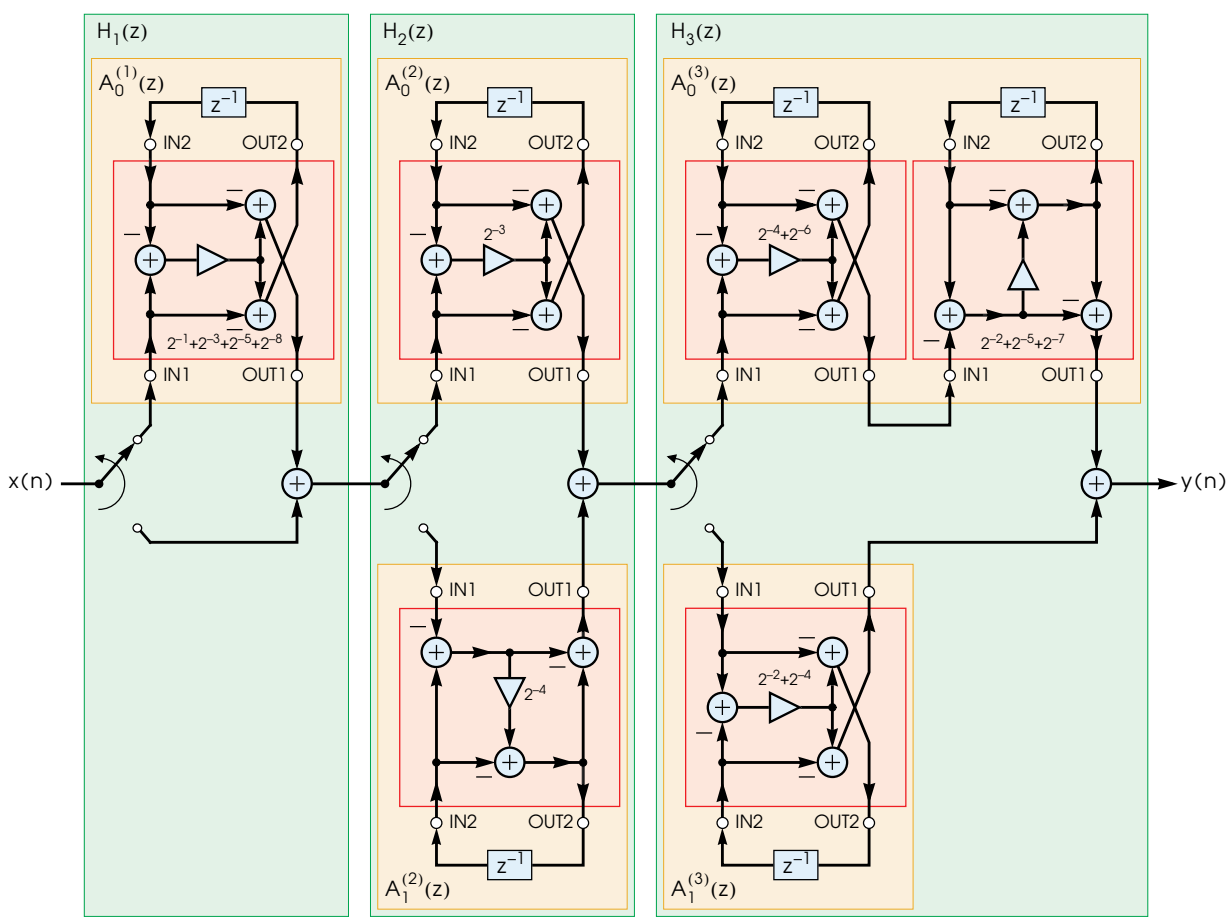

Fig. 18. An efficient implementation for the optimized finite-precision three-stage eighth-band decimator in Example 3.

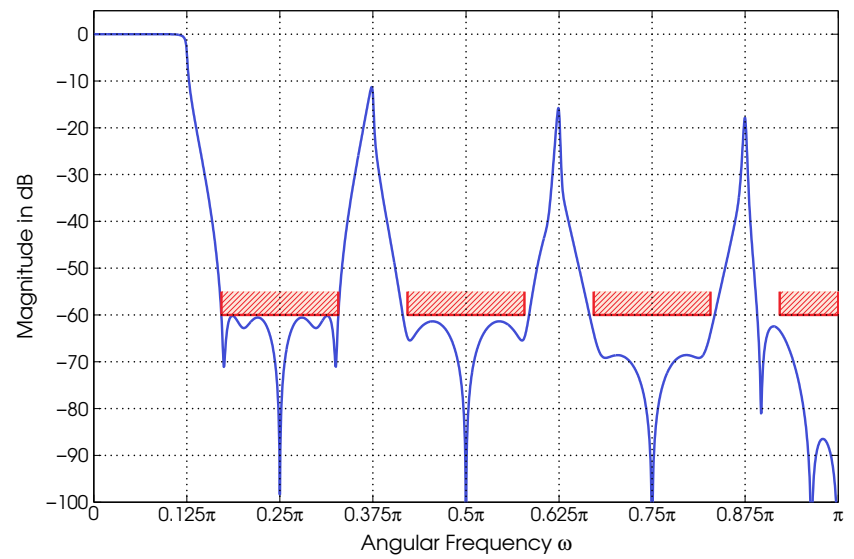

Fig. 19. Magnitude response for the optimized finite-precision single-stage eighth-band decimator in Example 3.

Antoniou, A. (1993). Digital Filters: Analysis, Design, and Applications, 2nd edn, McGraw-Hill. 


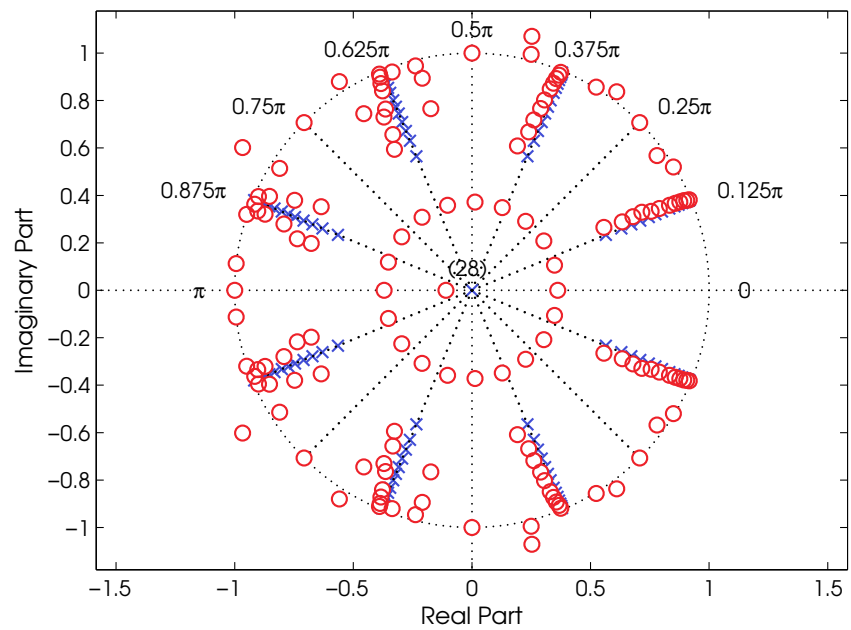

Fig. 20. Pole-zero plot for the optimized finite-precision single-stage eighth-band decimator in Example 3.

Bregović, R. (2003). Optimal design of perfect-reconstruction and nearly perfect-reconstruction multirate filter banks, Dr. Tech. dissertation, Dept. of Inform. Tech., Tampere Univ. of Tech, Finland, Tampere, Finland.

Brophy, F. J. \& Salazar, A. C. (1975). Two design techniques for digital phase network, Bell System Technical J. 54: 767-781.

Coleman, T., Branch, M. A. \& Grace, A. (1999). Optimization Toolbox User's Guide, The MathWorks, Inc. Version 2.

Crochiere, R. E. \& Rabiner, L. R. (1983). Multirate Digital Signal Processing, NJ: Prentice-Hall, Englewood Cliffs.

Deczky, A. G. (1972). Synthesis of recursive digital filters using the minimum- $p$ error criterion, IEEE Trans. Audio Electroacoust. AU-20: 257-263.

Dutta, S. R. K. \& Vidyasagar, M. (1977). New algorithms for constrained minimax optimization, Math. Program. 13: 140-155.

Fettweis, A. (1986). Wave digital filters: Theory and practice, Proc. IEEE 74: 270-327.

Fettweis, A., Levin, H. \& Sedlmeyer, A. (1974). Wave digital lattice filters, Int. J. Circuit Theory Appl. 2(2): 203-211.

Földvári-Orosz, J., Henk, T. \& Simonyi, E. (1991). Simultaneous amplitude and phase approximation for lumped and sampled filters, Int. J. Circuit Theory Appl. 19: 77-100.

Gazsi, L. (1985). Explicit formulas for lattice wave digital filters, IEEE Trans. Circuits Syst. CAS-32(1): 68-88.

Herrmann, O., Rabiner, L. R. \& Chan, D. S. (1973). Practical design rules for optimum finite impulse response lowpass digital filters, Bell Syst. Tech. J. 52(6): 769-799.

Jaworski, B. \& Saramäki, T. (1994). Linear phase IIR filters composed of two parallel allpass sections, Proc. IEEE Int. Symp. Circuits Syst., London, England, pp. 537-540.

Jones, A., Lawson, S. \& Wicks, T. (1991). Design of cascaded allpass structures with magnitude and delay constraint using simulated annealing and quasi-Newton methods, Proc. IEEE Int. Symp. Circuits Syst., Vol. 5, Singapore, pp. 2439-2442. 
Lawson, S. \& Wicks, T. (1992). Design of efficient digital filters satisfying arbitrary loss and delay specifications, Proc. Inst. Elect. Eng., Pt. G 139: 611-620.

Leeb, F. (1991). Lattice wave digital filters with simultaneous conditions on amplitude and phase, Proc. IEEE Int. Conf. Acoustics, Speech, and Signal Processing, Toronto, Canada, pp. 1645-1648.

Milić, L. D. \& Lutovac, M. D. (1999). Design of multiplierless elliptic IIR filters with a small quantization error, IEEE Trans. Signal Processing 47: 469-479.

Ohlsson, H., Gustafsson, O. \& Wanhammar, L. (2001). Arithmetic transformations for increased maximal sample rate of bit-parallel bireciprocal lattice wave digital filters, Proc. IEEE Int. Symp. Circuits Syst., Sydney, Australia.

Rabiner, L. \& Gold, B. (1975). Theory and Application of Digital Signal Processing, Englewood Cliffs, NJ: Prentice-Hall.

Regalia, P. A. (1993). Special filter design, in S. K. Mitra \& J. F. Kaiser (eds), Handbook for Digital Signal Processing, John Wiley and Sons, New York, chapter 13, pp. 907-980.

Regalia, P. A., Mitra, S. K. \& Vaidyanathan, P. P. (1988). The digital all-pass filter: A versatile signal processing building block, Proc. IEEE 76(1): 19-37.

Renfors, M. \& Saramäki, T. (1986). A class of approximately linear phase digital filters composed of allpass subfilters, Proc. IEEE Int. Symp. Circuits Syst., San Jose, CA, pp. 678681.

Renfors, M. \& Saramäki, T. (1987). Recursive Nth-band digital filters - Part I: Design and properties; Part II: Design of multistage decimators and interpolators, IEEE Trans. Circuits Syst. CAS-34(1): 24-51.

Renfors, M. \& Zigouris, E. (1988). Signal processor implementation of digital all-pass filters, IEEE Trans. Acoust., Speech, Signal Processing 36: 714-729.

Saramäki, T. (1985). On the design of digital filters as a sum of two all-pass filters, IEEE Trans. Circuits Syst. CAS-32(11): 1191-1193.

Saramäki, T. (1993). Finite impulse response filter design, in S. K. Mitra \& J. F. Kaiser (eds), Handbook for Digital Signal Processing, New York: John Wiley and Sons, chapter 4, pp. 155-277.

Saramäki, T. \& Bregović, R. (2002). Multirate systems and filter banks, in G. Jovanovic-Dolecek (ed.), Multirate Systems: Design E Applications, Hershey: Idea Group Publishing, chapter II, pp. 27-85.

Saramäki, T. \& Renfors, M. (1987). A novel approach for the design of IIR filters as a tapped cascaded interconnection of identical allpass subfilters, Proc. IEEE Int. Symp. Circuits Syst., Philadelphia, PA, pp. 629-632.

Saramäki, T. \& Renfors, M. (1995). A Remez-type algorithm for designing digital filters composed of all-pass sections based on phase approximations, Proc. 38th Midwest Symp. Circuits Syst., Rio de Janeiro, Brazil, pp. 571-575.

Saramäki, T. \& Renfors, M. (1998). Nth-band filter design, Proc. IX European Signal Processing Conf., Island of Rhodes, Greece, pp. 1943-1947.

Saramäki, T. \& Ritoniemi, T. (1993). Optimization of digital filter structures for VLSI implementation, Automatica 34: 111-116.

Saramäki, T. \& Yli-Kaakinen, J. (2002). Design of digital filters and filter banks by optimization: Applications, Technical Report No. 15, Tampere International Center for Signal Processing. 119 pages.

Schüßler, H. (2010). Digitale Signalverarbeitung 2, Springer-Verlag, Berlin. 
Surma-aho, K. (1997). Design of approximately linear-phase recursive filters, Master's thesis, Dept. of Electr. Eng., Tampere Univ. of Tech., Finland.

Surma-aho, K. \& Saramäki, T. (1999). A systematic technique for designing approximately linear phase recursive digital filters, IEEE Trans. Circuits Syst. II 46(7): 956-962.

Taxén, L. (1981). Polyphase filter banks using wave digital filters, IEEE Trans. Acoust., Speech, Signal Processing ASSP-29: 423-428.

Vollmer, M. \& Kopmann, H. (2002). A novel approach to an IIR digital filter bank with approximately linear phase, Proc. IEEE Int. Symp. Circuits Syst., Vol. II, Scottsdale, Arizona, pp. 512-515.

Wanhammar, L. (1998). DSP Integrated Circuits, New York: Academic.

Yli-Kaakinen, J. (1998). Optimization of recursive digital filters for practical implementation, Dipl. Eng. thesis, Dept. of Elect. Eng., Tampere Univ. of Tech., Finland.

Yli-Kaakinen, J. (2002). Optimization of digital filters for practical implementations, Dr. Tech. dissertation, Dept. of Inform. Tech., Tampere Univ. of Tech., Finland.

Yli-Kaakinen, J., Kupiainen, T., Hu, M., Uusikartano, R. \& Renfors, M. (1999). Multirate digital filter design for a PAL TV modulator, IEEE Trans. Consumer Electron. 45(3): 970-974.

Yli-Kaakinen, J. \& Saramäki, T. (1999a). Design of very low-sensitivity and low-noise recursive filters using a cascade of low-order lattice wave digital filters, IEEE Trans. Circuits Syst. II 46(7): 906-914.

Yli-Kaakinen, J. \& Saramäki, T. (1999b). An efficient algorithm for the design of lattice wave digital filters with short coefficient wordlength, Proc. IEEE Int. Symp. Circuits Syst., Vol. III, Orlando, FL, pp. 443-448.

Yli-Kaakinen, J. \& Saramäki, T. (2000). An algorithm for the design of multiplierless approximately linear-phase lattice wave digital filters, Proc. IEEE Int. Symp. Circuits Syst., Vol. 2, Geneva, Switzerland, pp. 77-80.

Yli-Kaakinen, J. \& Saramäki, T. (2005). A systematic algorithm for designing multiplierless computationally efficient recursive decimators and interpolators, Proc. 2005 IEEE Int. Symp. Image and Signal Process. Analysis, Zagreb, Croatia, pp. 167-172.

Yli-Kaakinen, J. \& Saramäki, T. (2007). A systematic algorithm for the desing of lattice wave digital filters with short-coefficient wordlength, IEEE Trans. Circuits Syst. I 54(8): 1838-1851. 
www.intechopen.com 


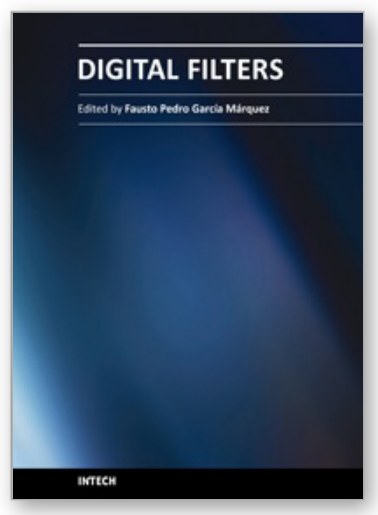

\author{
Digital Filters \\ Edited by Prof. Fausto Pedro GarcÃa MÃ $i r q u e z$
}

ISBN 978-953-307-190-9

Hard cover, 290 pages

Publisher InTech

Published online 11, April, 2011

Published in print edition April, 2011

The new technology advances provide that a great number of system signals can be easily measured with a low cost. The main problem is that usually only a fraction of the signal is useful for different purposes, for example maintenance, DVD-recorders, computers, electric/electronic circuits, econometric, optimization, etc. Digital filters are the most versatile, practical and effective methods for extracting the information necessary from the signal. They can be dynamic, so they can be automatically or manually adjusted to the external and internal conditions. Presented in this book are the most advanced digital filters including different case studies and the most relevant literature.

\title{
How to reference
}

In order to correctly reference this scholarly work, feel free to copy and paste the following:

Juha Yli-Kaakinen and Tapio Saramäki (2011). A Systematic Algorithm for the Synthesis of Multiplierless Lattice Wave Digital Filters, Digital Filters, Prof. Fausto Pedro GarcÃa MÃirquez (Ed.), ISBN: 978-953-307190-9, InTech, Available from: http://www.intechopen.com/books/digital-filters/a-systematic-algorithm-for-thesynthesis-of-multiplierless-lattice-wave-digital-filters

\section{INTECH}

open science | open minds

\section{InTech Europe}

University Campus STeP Ri Slavka Krautzeka 83/A 51000 Rijeka, Croatia

Phone: +385 (51) 770447

Fax: +385 (51) 686166 www.intechopen.com

\section{InTech China}

Unit 405, Office Block, Hotel Equatorial Shanghai No.65, Yan An Road (West), Shanghai, 200040, China 中国上海市延安西路65号上海国际贵都大饭店办公楼 405 单元 Phone: +86-21-62489820

Fax: $+86-21-62489821$ 
(C) 2011 The Author(s). Licensee IntechOpen. This chapter is distributed under the terms of the Creative Commons Attribution-NonCommercialShareAlike-3.0 License, which permits use, distribution and reproduction for non-commercial purposes, provided the original is properly cited and derivative works building on this content are distributed under the same license. 